UNIVERSIDADE DE SÃO PAULO

ESCOLA DE ARTES, CIÊNCIAS E HUMANIDADES PRGRAMA DE PÓS-GRADUAÇÃO EM CIÊNCIAS DA ATIVIDADE FÍSICA

PAULO TOSHIO UCHIYAMA

\title{
AVALIAÇÃO DO CONTROLE POSTURAL UNIPODAL APÓS UMA MANOBRA DE MANIPULAÇÃO CERVICAL
}




\title{
PAULO TOSHIO UCHIYAMA
}

\section{AVALIAÇÃO DO CONTROLE POSTURAL UNIPODAL APÓS UMA MANOBRA DE MANIPULAÇÃO CERVICAL}

\author{
Versão original
}

Dissertação apresentada à Escola de Artes, Ciências e Humanidades da Universidade de São Paulo para obtenção do título de Mestre em Ciências pelo Programa de PósGraduação em Ciências da Atividade Física.

Área de Concentração: Atividade Física, Saúde e Lazer.

Orientador: Prof. Dr. Fernando Henrique Magalhães. 
Autorizo a reprodução e divulgação total ou parcial deste trabalho, por qualquer meio convencional ou eletrônico, para fins de estudo e pesquisa, desde que citada a fonte.

Uchiyama, Paulo Toshio

Avaliação do controle postural unipodal após uma manobra de manipulação cervical / Paulo Toshio Uchiyama, orientador, Fernando Henrique Magalhães. - São Paulo, 2016

$48 \mathrm{f} .:$ il.

Dissertação (Mestrado em Ciências) - Programa de PósGraduação em Ciências da Atividade Física, Escola de Artes, Ciências e Humanidades, Universidade de São Paulo, São Paulo.

Versão original

1. Postura - Manipulação. 2. Postura - Terapia. 3.

Desempenho motor. 4. Controle motor. 5. Região cervical. I. Magalhães, Fernando Henrique, orient. II. Título

CDD 22.ed. - 615.82 


\section{FOLHA DE APROVAÇÃO}

\section{Paulo Toshio Uchiyama}

\section{Avaliação do controle postural unipodal após uma manobra de manipulação cervical}

Dissertação apresentada à Escola de Artes, Ciências e Humanidades da Universidade de São Paulo para obtenção do título de Mestre em Ciências pelo Programa de PósGraduação em Ciências da Atividade Física.

Área de Concentração: Atividade Física, Saúde e Lazer.

Aprovado em: / / 2016.

\section{Banca Examinadora}

Prof. Dr.

Julgamento:

Prof. Dr.

Julgamento:

Prof. Dr.

Julgamento:
Instituição:

Assinatura:

Instituição:

Assinatura:

Instituição:

Assinatura: 


\section{RESUMO}

UCHIYAMA, Paulo Toshio. Avaliação do controle postural unipodal após uma manobra de manipulação cervical. 2016. 44 f. Dissertação (Mestrado em Ciências) - Escola de Artes, Ciências e Humanidades, Universidade de São Paulo, São Paulo, 2016. Versão original.

O ser humano, durante a postura ortostática, apresenta oscilações posturais aleatórias em relação à vertical que são controladas pelo sistema nervoso central com base em informações sensoriais provenientes dos sistemas visual, vestibular e somatossensorial. Mecanoreceptores e fusos neuromusculares localizados na coluna cervical transmitem informações aferentes tanto para o sistema vestibular quanto para o sistema proprioceptivo, contribuindo significativamente para controle postural. Vários autores têm proposto intervenções baseadas em terapia manual, sugerindo que manobras de manipulação da coluna vertebral podem ser benéficas para o tratamento da tontura e vertigem de origem cervical. No entanto, a maioria desses estudos tem focado em sintomas gerais como medidas clínicas de dor, severidade do acometimento, ocorrência de episódios agudos, etc. Portanto, fica clara a necessidade de um estudo acerca dos efeitos de uma manobra de manipulação vertebral, em nível cervical, sobre o controle postural de sujeitos saudáveis. A hipótese do presente estudo foi que uma liberação das estruturas cervicais (causada pela manobra de manipulação) geraria uma melhora no influxo de informações sensoriais, e, consequentemente, uma melhora no sistema de controle postural. Sete sujeitos foram instruídos a permanecer, durante 60 segundos, sobre uma plataforma de força, o mais quietos possível, em apoio unipodal, proporcionando assim medidas extraídas do sinal da trajetória do centro de pressão, do centro de massa e variações na posição angular das articulações do tornozelo, joelho e quadril. Cada sujeito participou de duas sessões experimentais, sendo que em uma delas realizou testes de equilíbrio antes e depois da manobra de manipulação cervical (sessão experimental, EXP) e no outro dia realizou os mesmos testes de equilíbrio antes e depois de uma intervenção placebo (sessão controle, CTRL). Os resultados sugerem um efeito agudo da manobra de manipulação cervical, já que foi observada redução das oscilações posturais durante as primeiras tentativas realizadas após a intervenção. Nas sessões CTRL, o mesmo efeito não foi observado, pois o sistema de controle postural se mostrou estável durante todo o experimento.

Palavras-chave: postura, manipulação, pescoço, músculo, vértebra, desempenho motor, terapia manual. 


\begin{abstract}
UCHIYAMA, Paulo Toshio. Evaluation of unipodal postural control after a cervical manipulation maneuver. 2016. 44 p. Dissertation (Master of Science) - School of Arts, Sciences and Humanities, University of São Paulo, São Paulo, 2016. Original version.

Human subjects during upright stance show a random postural sway with respect to vertical, which is controlled by the central nervous system on the basis of information from sensory inputs applied to the visual, vestibular and proprioceptive systems. Mechanoreceptors and muscle spindles in the cervical spine provide afferent information for both the vestibular and the proprioceptive systems, thereby contributing to postural control. Several authors have proposed interventions based on manual therapy, suggesting that manipulation of the spine can be beneficial for treating dizziness and vertigo of cervical origin. However, few studies have investigated the effect of the spinal manipulation on postural control, as most have focused on more general symptoms such as clinical measures of pain, severity and occurrence of acute episodes, etc. Given the importance of the structures of the neck for controlling upright stance, there is a clear need for a study on the effects of cervical spinal manipulation on postural control of healthy subjects. The hypothesis of this work was that a release of the cervical structures (caused by cervical manipulation) would enhance the influx of sensory information, thereby improving postural control. Seven subjects were asked to remain for 60 seconds on a force platform, as quiet as possible, on single-leg stance. Measurements associated with the center of pressure and center of mass trajectories were assessed, as well as information about the ankle, knee and hip positions (i.e. angle variation). Two experimental conditions were tested: before ( 5 trials) and after ( 5 trials) cervical manipulation. Each subject participated in two experimental sessions, the first one with balance tests before and after the cervical manipulation (experimental session, EXP) and the other one with the same balance tests before and after a placebo intervention (control session, CTRL). The results suggest an acute effect of the cervical manipulation, as reduced postural sway was observed during the first trials after the intervention. Such an effect was not observed in the CTRL sessions, as the postural control system remained stable throughout the experiment.
\end{abstract}

Key words: posture, manipulation, neck, spine, motor performance, manual therapy. 


\section{LISTA DE ILUSTRAÇÕES}

Figura 1: Trechos correspondentes a 100 segundos de sinais relativos ao deslocamento do centro de pressão nos planos sagital (CoPap) e frontal (CoPml). As linhas horizontais mostradas nos gráficos da direita representam o valor RMS do CoP, medida comumente utilizada para medir a amplitude das oscilações posturais. Figura adaptada de (MAGALHAES; KOHN, 2011).... 12

Figura 2: Rota das artérias vertebrais através da coluna cervical. Figura adaptada de (KERRY; TAYLOR, 2006).

Figura 3: Posicionamento dos marcadores (emissores de infravermelho) e diagrama dos 4 ângulos segmentares no plano sagital: $\theta$ Tornozelo; $\theta$ Joelho; $\theta$ Quadril.

Figura 4: Esquema do arranjo experimental utilizado nas avaliações posturais, com o sujeito posicionado sobre a plataforma de força em apoio unipodal e sobre o bloco de espuma. Durante todas as condições experimentais os sujeitos utilizaram um fone de ouvido, evitando que ruídos sonoros do ambiente do laboratório interferissem nas avaliações. Os marcadores do sistema de aquisição de imagem não estão representados nessa figura.

Figura 5: Procedimentos para manobra de manipulação de C7 sobre $\mathrm{T} 1$, em rotação direita e inclinação direita (RdSd), vista de dois ângulos diferentes. Figura adaptada de (CUECO, 2008).

Figura 6: Quadrantes superiores representam os valores individuais (normalizados) para o parâmetro “Área” computado do sinal do CoP. Cada sujeito está representado com uma cor diferente. Em cada quadro, os cinco primeiros valores (á esquerda) correspondem ás tentativas realizadas antes da intervenção (manobra de manipulação cervical), enquanto os cinco valores posteriores (à direita) correspondem ás tentativas realizadas após a intervenção. O quadro da esquerda mostra valores obtidos durante a sessão experimental (EXP), na qual a manobra de manipulação cervical foi realizada, enquanto o quadro da direita representa a sessão controle (CTRL), na qual uma intervenção "placebo" foi aplicada. Nos quadrantes inferiores, os símbolos representam a média do grupo e as linhas verticais representam o desvio padrão amostra $(n=7)$. Asteriscos indicam diferenças significativas para a média observada nas repetições indicadas em comparação às demais.

Figura 7: Idêntico á Figura 6, porém para o parâmetro "RMSap" computado do sinal do CoP. 29

Figura 8: Idêntico á Figura 6, porém para o parâmetro "RMSml" computado do sinal do CoP 30 
Figura 9: Idêntico á Figura 6, porém para o parâmetro "VMap" computado do sinal do CoP. 31

Figura 10: Idêntico á Figura 6, porém para o parâmetro "VMml" computado do sinal do CoP 32

Figura 11: Idêntico á Figura 6, porém para o parâmetro " $\theta$ Tornozelo" computado a partir dos sinais da posição dos marcadores (cinemática). 35

Figura 12: Idêntico á Figura 6, porém para o parâmetro " $\theta$ Joelho" computado a partir dos sinais da posição dos marcadores (cinemática). 36

Figura 13: Idêntico á Figura 6, porém para o parâmetro " $\theta$ Joelho" computado a partir dos sinais da posição dos marcadores (cinemática). 


\section{LISTA DE TABELAS}

Tabela 1: Resultados da ANOVA de duas vias (medidas repetidas) para os parâmetros do CoP 33

Tabela 2: Resultados da ANOVA de duas vias (medidas repetidas) para os parâmetros do COM. ..... 34

Tabela 3: Resultados da ANOVA de duas vias (medidas repetidas) para os parâmetros de variação nas posições angulares ( $\theta$ Tornozelo, $\theta$ Joelho e $\theta$ Quadril). 38 


\section{LISTA DE ABREVIATURAS E SIGLAS}

ANOVA: Análise de Variância

AP: Ântero-Posterior

cm: centímetro(s)

CoP: Centro de Pressão

COM: Centro de Massa

CTRL: Controle

EXP: Experimental

mm: milímetro(s)

$\mathbf{N}$ : Newton(s)

RMS: Root Mean Square (valor eficaz)

RMSap: Root Mean Square (valor eficaz) para a direção ântero-posterior

RMSml: Root Mean Square (valor eficaz) para a direção médio-lateral

s: $\operatorname{segundo}(\mathrm{s})$

VM: Velocidade Média

VMap: Velocidade Média para a direção ântero-posterior

VMml: Velocidade Média para a direção médio-lateral 


\section{Sumário}

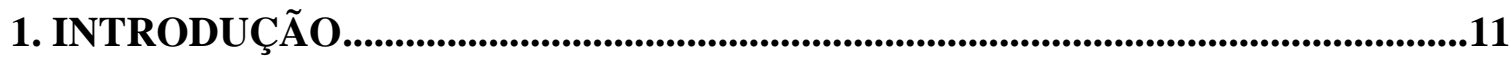

1.1 Controle Postural e a Influência das Informações Sensoriais .........................11

1.2 Estruturas Cervicais e sua Relação com o Controle Postural ...........................13

1.3 Efeito da Terapia Manual no Controle Postural e no Tratamento de

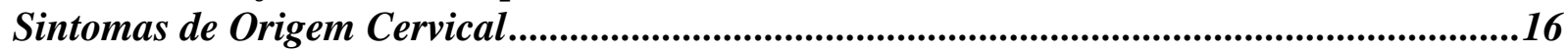

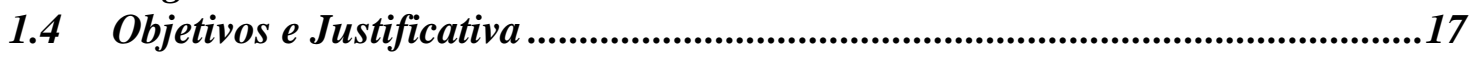

2. METOLOLOGIA .............................................................................................................19

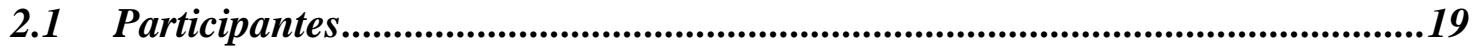

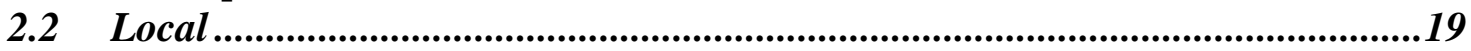

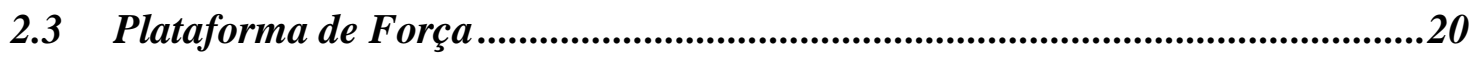

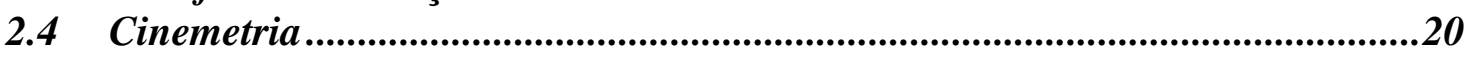

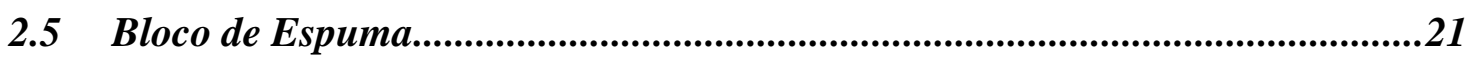

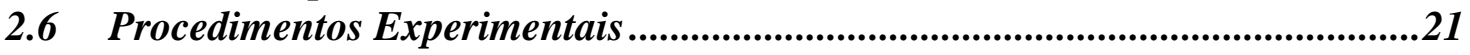

2.6.1 Manobra de manipulação cervical ..........................................................23

2.7 Processamento e Análise dos Sinais.........................................................24

2.7.1 Sinais da plataforma de Força (cinética) ....................................................2. 25

2.7.2 Sinais do sistema de aquisição de imagens (cinemática) ..............................26

2.7.3 Análise Estatística...................................................................................26

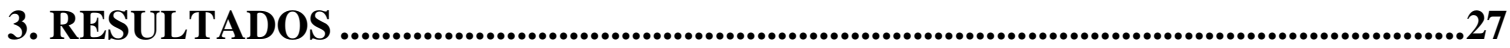

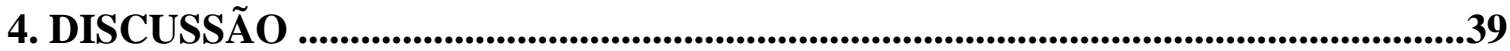

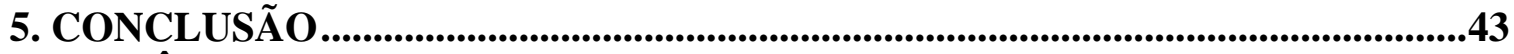

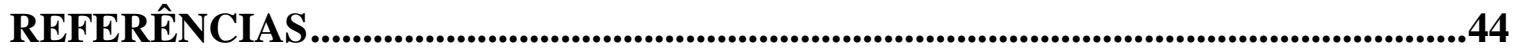




\section{INTRODUÇÃO}

\subsection{Controle Postural e a Influência das Informações Sensoriais}

O controle postural é uma função complexa que envolve a manutenção da projeção vertical do centro de gravidade do corpo dentro de uma base de suporte estabelecida pelo contato (apoio) dos pés sobre o solo. Devido à variabilidade intrínseca de diversos mecanismos fisiológicos (e.g., atividade cortical e de outros centros supraespinhais, disparo de unidades motoras, contrações musculares fásicas, etc...) (WATANABE et al., 2013), o ser humano, durante a postura ortostática, exibe uma oscilação postural aleatória que tem sido investigada por pesquisadores de várias áreas (BOTTARO et al., 2005; DUARTE; ZATSIORSKY, 2000; MAGALHAES; KOHN, 2011; MAURER et al., 2005; PETERKA, 2000; ZATSIORSKY; DUARTE, 2000). Essa oscilação é controlada pelo sistema nervoso central com base em informações oriundas de entradas sensoriais dos sistemas visual, vestibular e somatosensorial (SHUMWAY-COOK; WOOLLACOTT, 2001). Um déficit em qualquer um desses sistemas sensoriais implica em um prejuízo no desempenho do sistema de controle postural, o que causa um aumento na amplitude das oscilações posturais e uma maior propensão a quedas.

A forma mais comumente empregada para mensurar essa oscilação postural é por meio de uma plataforma de força. Esta mede as forças e momentos em três direções ortogonais, das quais se calcula o centro de pressão (CoP) (ver Figura 1 para exemplo), que é o ponto na superfície de apoio onde é aplicada a força resultante de reação do solo. Várias abordagens têm sido propostas para a quantificação das características do sinal aleatório associado ao CoP (DUARTE; ZATSIORSKY, 2001; MASANI et al., 2003; PRIETO et al., 1996; ZATSIORSKY; DUARTE, 2000). 

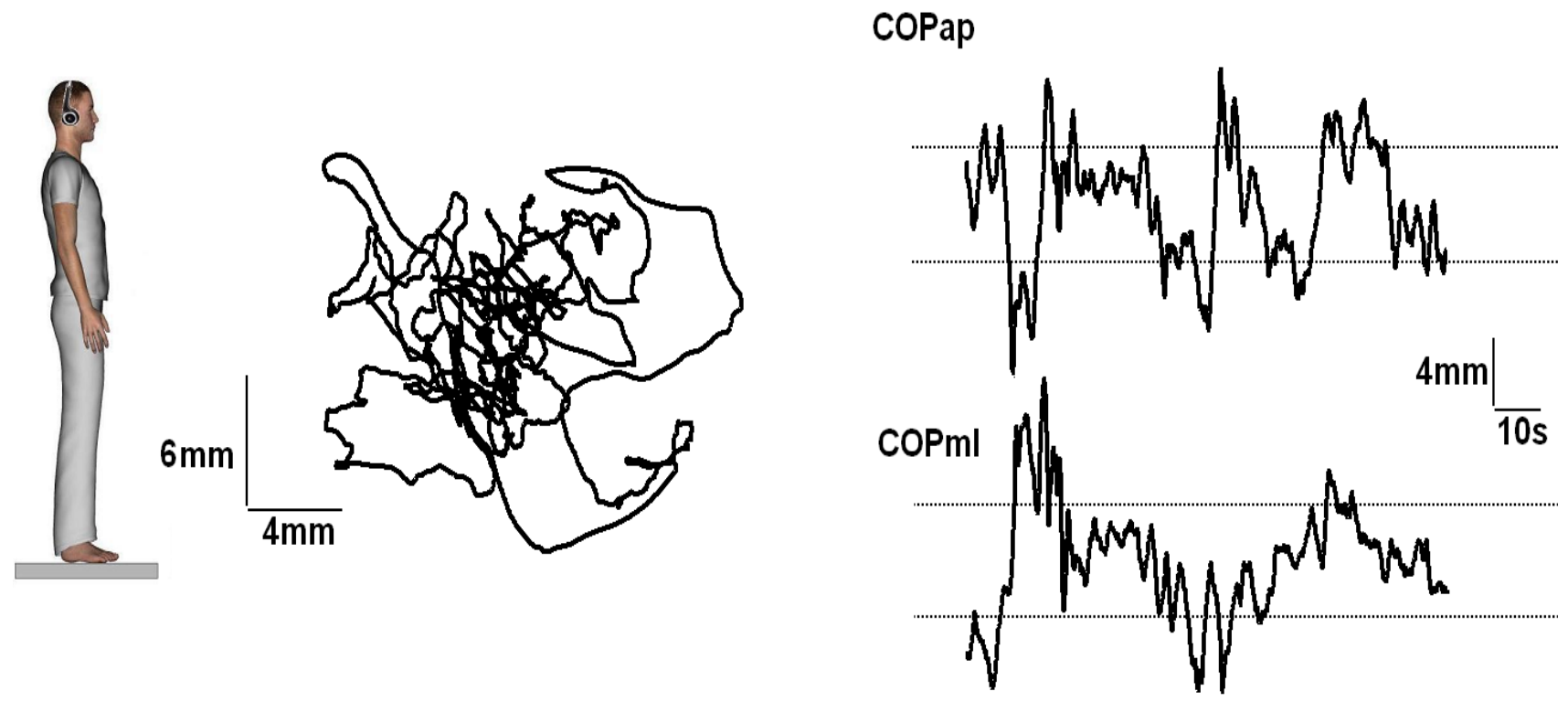

Figura 1: Trechos correspondentes a 100 segundos de sinais relativos ao deslocamento do centro de pressão nos planos sagital (CoPap) e frontal (CoPml). As linhas horizontais mostradas nos gráficos da direita representam o valor RMS do CoP, medida comumente utilizada para medir a amplitude das oscilações posturais. Figura adaptada de (MAGALHAES; KOHN, 2011).

Sendo assim, diversos estudos têm sido realizados para avaliar o controle postural de sujeitos sadios ou com patologias, por meio da análise de parâmetros associados ao CoP. Além dos estudos das oscilações posturais sem a aplicação de perturbações ao sujeito experimental, a manipulação de entradas sensoriais como visuais, auditivas, proprioceptivas ou táteis, por exemplo, tem sido empregada na exploração de como o sistema nervoso humano se comporta na tarefa de manutenção da postura ereta quieta (EASTON et al., 1998; JEKA, 1997; PRIPLATA et al., 2003; WIERZBICKA et al., 1998).

Por exemplo, um toque efetuado pela ponta do dedo indicador sobre uma superfície externa rígida (a força exercida é mínima, não configurando um apoio mecânico) diminui consideravelmente a oscilação postural (JEKA, 1997; JEKA; LACKNER, 1994), o que indica que as informações sensoriais adquiridas pelos receptores táteis da ponta do dedo (i.e. relacionadas com as forças de contato entre o dedo e a superfície de apoio) fornecem ao sistema nervoso central informações relevantes que ajudam a manter a estabilidade postural. Por outro lado, a privação de informações sensoriais relevantes (e.g. visão, propriocepção) tende a causar uma piora na estabilidade postural, como evidenciado pelo aumento das oscilações posturais quando os sujeitos são instruídos a fechar os 
olhos durante a manutenção da postura (HAY et al., 1996) ou quando as fontes de informação proprioceptiva são perturbadas, como por exemplo pela aplicação de estímulos vibratórios sobre músculos e tendões de forma a "mascarar" as informações provenientes dos fusos neuromusculares (CAPICIKOVA et al., 2006). Outro exemplo é a manipulação das informações de origem vestibular através de correntes de baixas intensidades que, passando pelos processos mastoides, estimulam o sistema vestibular gerando aumento das oscilações posturais (DAY et al., 1997). Esse tipo de manipulação, chamada de estimulação vestibular galvânica, atua de forma a modular o padrão de disparos de potenciais de ação nas vias aferentes de origem vestibular (COURJON et al., 1987), produzindo assim um padrão não natural de informações vestibulares (MINOR; GOLDBERG, 1991), culminando em prejuízo para o sistema de controle postural.

Dessa forma, condições experimentais com manipulação das aferências sensoriais (e.g., olhos abertos e fechados, base de suporte rígida/maleável, adição/remoção de informações táteis, estimulação vestibular galvânica, etc.), têm sido utilizadas para investigar como o sistema de controle postural organiza as diferentes fontes de informações sensoriais, com resultados mostrando que diferentes "ênfases" ou "pesos" podem ser atribuídos aos diferentes sistemas sensoriais (HWANG et al., 2014), dependendo do tipo de tarefa postural, da idade do indivíduo, do nível de treinamento, ou em função de condições patológicas.

\subsection{Estruturas Cervicais e sua Relação com o Controle Postural}

Mecanoreceptores e fusos neuromusculares localizados na coluna cervical transmitem informações aferentes tanto para o sistema vestibular quanto para o sistema proprioceptivo. Os músculos intervertebrais profundos da coluna cervical possuem uma alta densidade de fusos neuromusculares, exercendo, portanto, um importante papel no controle da postura (ABRAHAMS, 1977; BOYD-CLARK et al., 2002; KULKARNI et al., 2001; LIU et al., 2003). Por sua vez, os mecanoreceptores das cápsulas articulares associadas às articulações facetárias cervicais são considerados importantes para a propriocepção (MCLAIN, 1994). De forma similar, o trato 
vestibuloespinhal conecta os fusos neuromusculares da região cervical com os núcleos vestibulares, influenciando significativamente o sistema de controle postural (BOLTON, 1998; NITZ; PECK, 1986).

Sabe-se que aferências cervicais estão envolvidas nos mecanismos de reflexo cérvico-cólico, cérvico-ocular e reflexo tônico do pescoço, os quais atuam em conjunto com outros reflexos que, regulados pelos sistemas visual e vestibular, contribuem para estabilização da cabeça, olhos e postura (TRELEAVEN, 2008). Dessa forma, as informações vestibulares e proprioceptivas são integradas de forma a estabelecer um panorama que nos permite determinar a posição e orientação do corpo no espaço (KARNATH, 1994). Mecanismos relacionados com alterações dessas estruturas cervicais podem estar associados aos sintomas de vertigem e de instabilidade postural observados em casos de lesão dos tecidos moles da região cervical (TRELEAVEN et al., 2008). Além disso, tais mecanismos são tidos como responsáveis pelas alterações de controle postural observadas em pacientes com quadro de dor crônica no pescoço e de atrofia dos músculos suboccipitais (ANDARY et al., 1998; HALLGREN et al., 1994; MCPARTLAND et al., 1997).

Evidências adicionais acerca da importância das estruturas cervicais para o controle postural têm sido demonstradas uma vez que injeções com anestesia aplicadas às raízes nervosas dorsais da coluna cervical superior podem induzir tontura e nistagmo (BIEMOND; DE JONG, 1969; COHEN, 1961; DE JONG et al., 1977), e estimulação elétrica nos músculos cervicais podem gerar sensação de inclinação e desequilíbrio (WAPNER et al., 1951). Como exemplo, Brandt e Bronstein (BRANDT; BRONSTEIN, 2001) abordaram um mecanismo no qual um quadro de dor no pescoço gerou mudanças nos padrões de disparo de receptores somatosensoriais localizados na região cervical, causando um desequilíbrio entre as informações vestibulares e musculares, resultando assim em vertigem de origem cervical. Estímulos vibratórios aplicados aos músculos do pescoço (i.e. musculatura cervical como trapézios e esternocleidomastoideos) alteram o padrão de disparados de potenciais de ação das aferentes sensoriais associadas aos fusos neuromusculares, causando alteração 
na percepção da posição da cabeça e do tronco (CEYTE et al., 2006), além de aumento das oscilações posturais (BOVE et al., 2009; GOMEZ et al., 2009).

Devamos ainda levar em consideração fatores relativos ao fluxo sanguíneo associado ao sistema arterial vertebrobasilar (MAGAREY et al., 2004), o qual é responsável por fornecer aporte sanguíneo para o tronco cerebral, bulbo, ponte, cerebelo e aparato vestibular, estruturas essas intimamente relacionadas ao controle do equilíbrio e da postura. Anatomicamente, as artérias vertebrais (direita e esquerda) originam-se das artérias subclaviculares e passam através dos forames transversos das vértebras cervicais de C6 até C1, e então entram no escalpo através do forame magno occipital (Figura 2). Dentro do escalpo, as duas artérias vertebrais (direita e esquerda) se juntam formando assim a artéria basilar. Nessa rota, alguns pontos críticos são notados, nos quais uma possível compressão desses vasos pode ocorrer (KERRY; TAYLOR, 2006). Mais especificamente, em vista das características anatômicas representadas na Figura 2, é facilmente notável que alterações sutis na postura da cabeça (e.g. rotação), nas estruturas ósseas ou nos tecidos moles adjacentes podem causar compressão dessas artérias (por exemplo, na altura dos forames transversos de C1 e C2 e da membrana atlanto-occipital). Nesse caso, uma redução do fornecimento sanguíneo pode causar sintomas como tontura, vertigem e perda de equilíbrio (além de outros sintomas como náusea, ataxia, nistagmo e perda de consciência) uma condição conhecida como "insuficiência arterial vertebrobasilar" (COMAN, 1986), que tem sido motivo de atenção por parte de terapeutas de diferentes áreas (KERRY; TAYLOR, 2006).

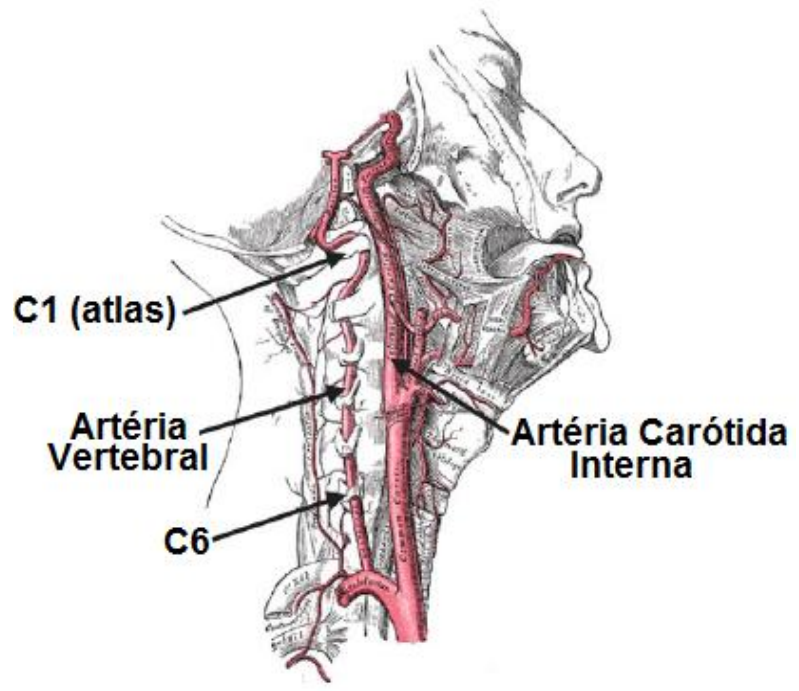

Figura 2: Rota das artérias vertebrais através da coluna cervical. Figura adaptada de (KERRY; TAYLOR, 2006). 


\subsection{Efeito da Terapia Manual no Controle Postural e no Tratamento de Sintomas de Origem}

\section{Cervical}

Grande parte das investigações acerca dos efeitos da terapia manual sobre o controle postural foram realizadas com foco em intervenções aplicadas sobre estruturas dos membros inferiores ou da coluna lombar [e.g. (ALBURQUERQUE-SENDIN et al., 2009; FERREIRA et al., 2007; GOERTZ et al., 2015; WASSINGER et al., 2014)], muito provavelmente por serem efetores diretamente relacionados á geração de forças que atuam de forma importante no controle de tarefas posturais. Por outro lado, o efeito de terapias manuais em nível cervical sobre o controle da postura tem recebido bem menos atenção, como comentado abaixo.

Vários autores têm proposto intervenções baseadas em terapia manual para o tratamento de tontura e desequilíbrio associados a sintomas na região cervical (BRANDT; BRONSTEIN, 2001; GALM et al., 1998; KARLBERG et al., 1996; ZHOU et al., 1999), sugerindo que manobras de manipulação da coluna vertebral podem ser benéficas para o tratamento da tontura e vertigem de origem cervical (FRAIX, 2010; GALM et al., 1998; HAWK et al., 2007; HEIKKILA et al., 2000; LOPEZ et al., 2011; LYSTAD et al., 2011). No entanto, duas recentes revisões sistemáticas da literatura concluem que ainda são limitadas as evidências que suportam o uso de terapia manual, em especial mobilização e manipulação vertebral, para o tratamento de tontura/vertigem de origem cervical (LYSTAD et al., 2011; REID; RIVETT, 2005). Além disso, poucos são os estudos que investigam diretamente o efeito dessas manobras sobre o controle postural, já que a maioria tem focado em sintomas mais gerais como medidas clínicas de dor, severidade do acometimento, ocorrência de episódios agudos, etc. (LYSTAD et al., 2011).

Em nossa revisão da literatura, foram encontrados apenas 3 estudos abordando o efeito da terapia manual em nível cervical sobre medidas diretamente relacionadas com o controle postural (FRAIX et al., 2013; KARLBERG et al., 1996; LOPEZ et al., 2011), sendo que todos esses estudos foram conduzidos em populações que já apresentavam alterações no sistema de controle postural. Mais especificamente, os estudos de (FRAIX et al., 2013) e (KARLBERG et al., 1996) envolveram 
amostras de sujeitos com sintomas de tontura e o estudo de (LOPEZ et al., 2011) foi conduzido em uma amostra de idosos. Nesse sentido, uma importante limitação desses estudos é que os mecanismos associados a essas alterações no sistema de controle postural não foram controlados de forma específica, visto que, tanto no caso da tontura como no caso dos idosos, o prejuízo sobre o sistema de controle postural (o que implica em maiores oscilações posturais) pode ter origens variadas, por exemplo, proprioceptivas ou vestibulares (LYSTAD et al., 2011; REID; RIVETT, 2005) no caso da tontura, e neural ou muscular no caso do envelhecimento (WOOLLACOTT et al., 1986). Sendo assim, não é possível inferir adequadamente acerca dos mecanismos associados aos efeitos da terapia manual sobre o controle postural, já que indivíduos com sintomas de diferentes origens podem ter feito parte da amostra. Além disso, os estudos citados anteriormente (FRAIX et al., 2013; KARLBERG et al., 1996; LOPEZ et al., 2011) não envolveram intervenções específicas restritas à apenas uma manobra de manipulação vertebral, mas mesclaram manobras de manipulação com outras intervenções como mobilizações lentas, liberação miofacial e articular, técnicas de manipulação craniana (FRAIX et al., 2013; LOPEZ et al., 2011), exercícios de estabilização to tronco e do pescoço, programas de treinamento domiciliar e modificações ergonômicas no trabalho (KARLBERG et al., 1996). Sendo assim, não é possível inferir sobre os efeitos/mecanismos de uma manobra específica, ficando esses estudos limitados a propor que o conjunto de terapias provavelmente tenha resolvido múltiplos desarranjos estruturais, gerando assim uma melhora na função global do sistema de controle neuromuscular.

\subsection{Objetivos e Justificativa}

Visto a importância das estruturas da região cervical no controle da postura, fica clara a necessidade de um estudo acerca dos efeitos de uma única manobra de manipulação vertebral, em nível cervical, sobre o controle postural de sujeitos saudáveis. Sendo assim, será possível avaliar o efeito de uma técnica específica de terapia manual sobre o controle postural, sem que variáveis como a presença de indivíduos sintomáticos ou assintomáticos dentro da amostra experimental interfira na 
interpretação dos resultados. A hipótese do presente trabalho é que uma liberação das estruturas cervicais (causada pela manobra de manipulação) gere, direta ou indiretamente, uma melhora no influxo de informações sensoriais, e, consequentemente, uma melhora no sistema de controle postural.

Além de promover um melhor entendimento da neurofisiologia do controle postural, os resultados a serem obtidos poderão indicar o potencial de uso clínico de uma manobra de manipulação cervical a fim de melhorar o controle postural de pessoas com diferentes acometimentos de origem cervical (vertigem, tontura, dor cervical, etc.).

Portanto, o objetivo do presente trabalho é investigar os efeitos de uma manobra de manipulação cervical sobre o controle postural de indivíduos saudáveis, através de medidas associadas aos sinais do $\mathrm{CoP}$ e do centro de massa (CoM) adquiridos durante uma tarefa de equilíbrio. 


\section{METOLOLOGIA}

\subsection{Participantes}

Foram coletados dados de 7 sujeitos com idade média de 28 anos. Os sujeitos participaram de duas sessões experimentais, em dias diferentes, sendo que em uma delas realizaram testes de equilíbrio antes e depois de uma manobra de manipulação cervical (sessão experimental, EXP) e no outro dia realizaram os mesmos testes de equilíbrio antes e depois de uma intervenção "placebo", na qual os sujeitos realizaram os mesmos procedimentos da sessão EXP, porém sem que a manobra de manipulação fosse executada efetivamente (sessão controle, CTRL). Como critério de inclusão, foi necessário que os indivíduos não apresentassem antecedentes prévios ou história atual de patologias neurológicas ou deformidades articulares. Os sujeitos assinaram termo de consentimento pósinformado para participarem dos experimentos.

\subsection{Local}

Os experimentos foram realizados no Laboratório de Engenharia Biomédica (LEB), que fica localizado na Escola Politécnica da Universidade de São Paulo. Neste laboratório, há equipamentos e experiência apropriados para a realização dos experimentos descritos aqui. Os atuais integrantes do laboratório são engenheiros biomédicos, fisioterapeutas e biólogos, provenientes tanto da pósgraduação em Neurociência e Comportamento (IP-USP), quanto da pós-graduação em Ciências da Atividade Física (EACH-USP). O pesquisador responsável (orientador) deste projeto pertence a ambos os programas de pós-graduação na USP. Todos os trabalhos com seres humanos realizados nesse laboratório passam pela avaliação e aprovação de um Comitê de Ética. 


\subsection{Plataforma de Força}

As informações sobre as 3 forças e 3 momentos nos eixos $\mathrm{X}, \mathrm{Y}$, e $\mathrm{Z}$ durante a tarefa postural foram adquiridas por meio de uma plataforma de força (AMTI, OR6-7-1000, Massachusetts, USA), totalizando 6 canais de aquisição.

\subsection{Cinemetria}

Dados cinemáticos foram obtidos por um sistema de captação de imagem Optotrak 3020 (Northern Digital, Inc., Waterloo, Ontario, Canada). Emissores de infravermelho foram posicionados: 1) no pé (cabeça do quinto metatarso direito); 2) no tornozelo (maléolo lateral direito); 3) no joelho (côndilo femoral lateral direito); 4) no quadril (trocânter maior direito) e; 5) no ombro (acrômio direito), como representado na Figura 3. O movimento dos emissores foi registrado por um conjunto de três câmeras posicionadas a aproximadamente $2.5 \mathrm{~m}$ à direita dos participantes.
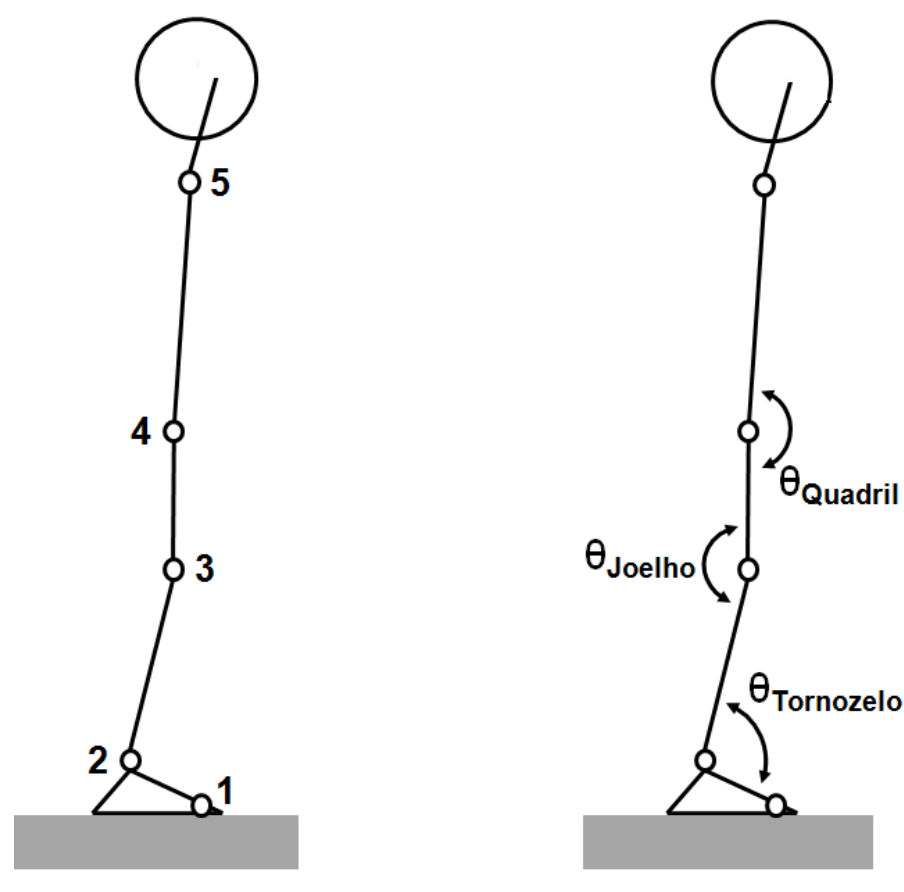

Figura 3: Posicionamento dos marcadores (emissores de infravermelho) e diagrama dos 4 ângulos segmentares no plano sagital: $\theta$ Tornozelo; $\theta$ Joelho; $\theta$ Quadril. 


\subsection{Bloco de Espuma}

Sabe-se que os pequenos movimentos contínuos e corretivos, exercidos durante a postura ereta quieta, conhecidos como oscilação postural, são iniciados por meio de mecanismos de feedback e feedforward coordenados pelos sistemas sensoriais e motores. Uma importante fonte dessas aferências é proveniente de mecanoreceptores cutâneos especializados presentes nas solas dos pés, que fornecem informações essenciais sobre as pressões exercidas na superfície de contato do pé com o solo. Para aumentar o desafio postural muitos trabalhos têm utilizado de métodos que adicionem alguma perturbação ao equilíbrio, tais como blocos de espuma (Foam Pad). Quando o sujeito é posicionado sobre uma superfície de espuma há uma diminuição da capacidade de detectar a maneira como a pressão dos pés está sendo distribuída, culminando em uma diminuição da orientação do corpo no espaço (HATTON et al., 2009; JANSSENS et al., 2013; PATEL et al., 2008; STANEK et al., 2013; WU; CHIANG, 1997).

Para aumentar a dificuldade das tarefas impostas durante a postura ereta quieta foi utilizado um bloco de espuma de borracha (Foam Pad, AIREX balance pad; 49,5 cm de comprimento, 40,5 $\mathrm{cm}$ de largura e $6,5 \mathrm{~cm}$ de altura) posicionado sobre a plataforma de força. O raciocínio por trás dessa escolha é que o bloco de espuma deve gerar um maior desafio ao sistema de controle postural além de diminuir o uso das informações sensoriais provenientes da sola dos pés para o controle da tarefa, aumentando assim a contribuição das aferências sensoriais relacionadas às estruturas cervicais para o controle do equilíbrio.

\subsection{Procedimentos Experimentais}

Os sujeitos foram instruídos a permanecer sobre a plataforma de força, o mais quietos possível, em apoio unipodal (i.e. com apenas o pé dominante tocando o solo), como representado na Figura 4. A tarefa postural em apoio unipodal foi escolhida por ser uma postura desafiadora, com maior exigência por parte do sistema vestibular e proprioceptivo em comparação á tarefas posturais 
em apoio bipedal. Duas condições experimentais foram testadas: antes e após uma manobra de manipulação cervical (descrita na seção 2.6.1). Vale ressaltar novamente que cada sujeito participou de duas sessões experimentais, em dias diferentes, sendo que em uma delas realizou testes de equilíbrio antes e depois da manobra de manipulação cervical (sessão experimental, EXP) e no outro dia realizou os mesmos testes de equilíbrio antes e depois de uma intervenção placebo, na qual os mesmos procedimentos da sessão EXP foram realizados, porém sem que a manobra de manipulação tivesse sido executada efetivamente (sessão controle, CTRL, ver detalhes a seguir). Cada sujeito realizou 5 repetições para cada condição experimental, sendo que cada repetição consistiu em permanecer durante $60 \mathrm{~s}$ na tarefa postural em apoio unipodal, sobre o bloco de espuma. A ordem de realização das sessões experimentais EXP e CTRL foi contrabalanceada entre os sujeitos, ou seja, aproximadamente metade dos sujeitos realizou a sessão EXP antes da sessão CTRL e a outra metade realizou primeiro a sessão EXP. Antes do início de cada sessão experimental, os participantes completaram 5 repetições de familiarização, com o intuito de minimizar qualquer efeito decorrente da prática.

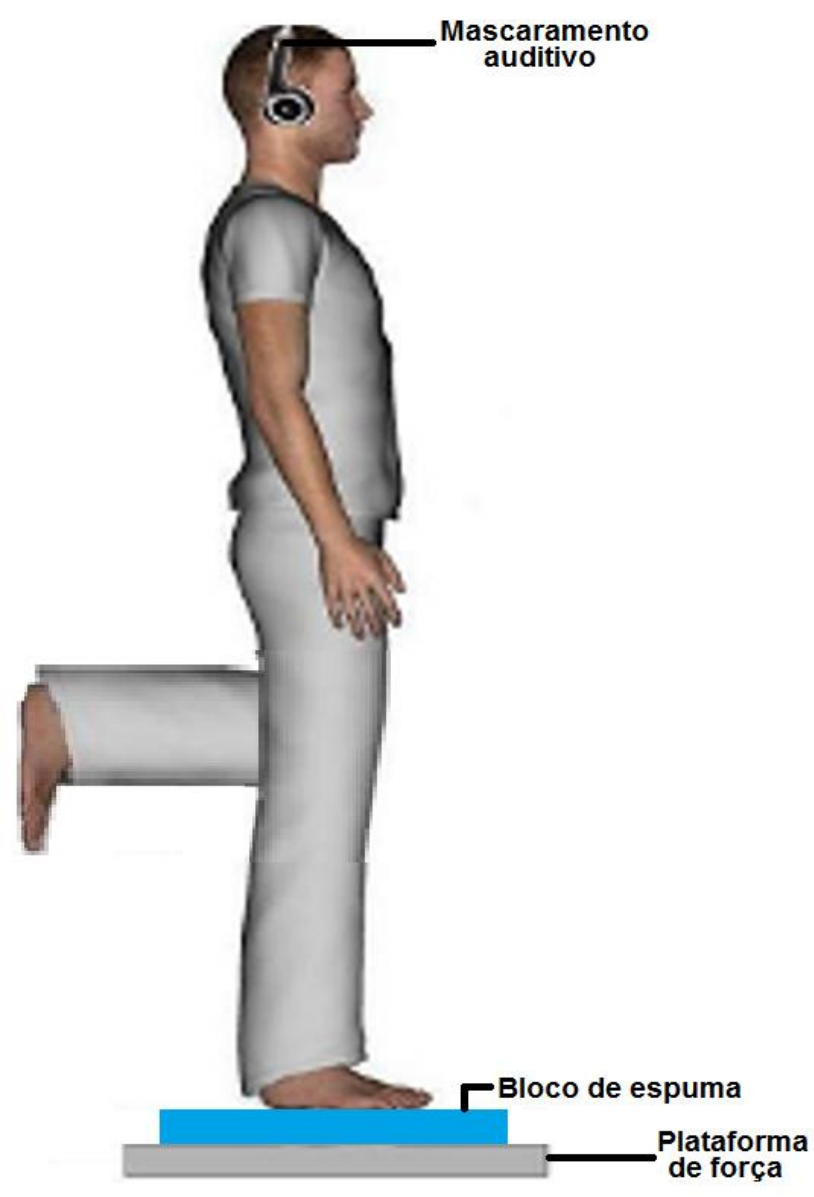

Figura 4: Esquema do arranjo experimental utilizado nas avaliações posturais, com o sujeito posicionado sobre a plataforma de força em apoio unipodal e sobre o bloco de espuma. Durante todas as condições experimentais os sujeitos utilizaram um fone de ouvido, evitando que ruídos sonoros do ambiente do laboratório interferissem nas avaliações. Os marcadores do sistema de aquisição de imagem não estão representados nessa figura. 


\subsubsection{Manobra de manipulação cervical}

A manobra de manipulação cervical foi realizada sempre pelo mesmo terapeuta, membro do laboratório e certificado como especialista em osteopatia, com mais de 10 anos de experiência prática. É importante ressaltar que o procedimento realizado sempre pelo mesmo terapeuta é desejável, de modo a reduzir a variabilidade entre uma intervenção e outra (FRAIX, 2010).

A manobra de manipulação realizada foi de C7 sobre T1, em rotação e inclinação, tanto á direita quanto à esquerda, ou seja, em rotação direita com inclinação direita (RdSd) e em rotação esquerda com inclinação esquerda (ReSe), assim como descrito em (CUECO, 2008). Tal manobra é comumente utilizada com o objetivo clínico de corrigir restrições de movimento de $\mathrm{C} 7$, liberando estruturas envolvidas nos movimentos de rotação e flexão-lateral. Esse nível escolhido (de C7 sobre T1) justifica-se por estudos anteriores mostrando a importância da inter-relação entre as vértebras cervicais e torácicas para a cinemática e função sensorial do pescoço (TSANG et al., 2013; WASSINGER et al., 2015).

Durante a manobra, o terapeuta assumiu a posição em pé, ao lado do participante. Por sua vez, o participante permaneceu em decúbito ventral (prono) com a cabeça em posição neutra, com ligeira flexão do pescoço (tal flexão é obtida ao posicionar um travesseiro abaixo do osso esterno do participante). O terapeuta então realizou os seguintes procedimentos: 1) o polegar (esquerdo ou direito, dependendo do lado da manobra) do terapeuta foi posicionado sobre a parte lateral (esquerda ou direita) da apófise espinhosa, por cima da borda superior do músculo trapézio (ver Figura 5 para exemplo de manobra em RdSd), enquanto o restante dos dedos dessa mão envolviam anteriormente a clavícula do participante; 2) esse mesmo antebraço do terapeuta foi orientado obliquamente na direção do impulso (manobra) em sentido ínfero-medial (na direção da axila do participante); 3) a outra mão do terapeuta foi posicionada sobre a região temporomandibular do participante, de forma a impor uma tensão em inclinação (Figura 5), até que o terapeuta percebesse que a apófise espinhosa de C7 fazia contato com o polegar de sua outra mão; e 4) a partir desta posição, foi realizado então um impulso rápido e vigoroso com polegar (o que geralmente gera um “estalido", ou “trust”), 
caracterizando a manobra de manipulação de forma propriamente dita. Diferentemente de situações clínicas, nas quais é comum que a manobra seja realizada ou à esquerda (ReSe), ou á direita (RdSd), com base em avaliação física prévia, para os fins dos experimentos descritos aqui os participantes foram, nas sessões EXP, submetidos ás duas manobras, uma á esquerda e uma á direita (sendo a ordem dos lados contrabalanceada entre os sujeitos). Já nas sessões CTRL, todos os procedimentos descritos aqui foram repetidos, com exceção do item 4.
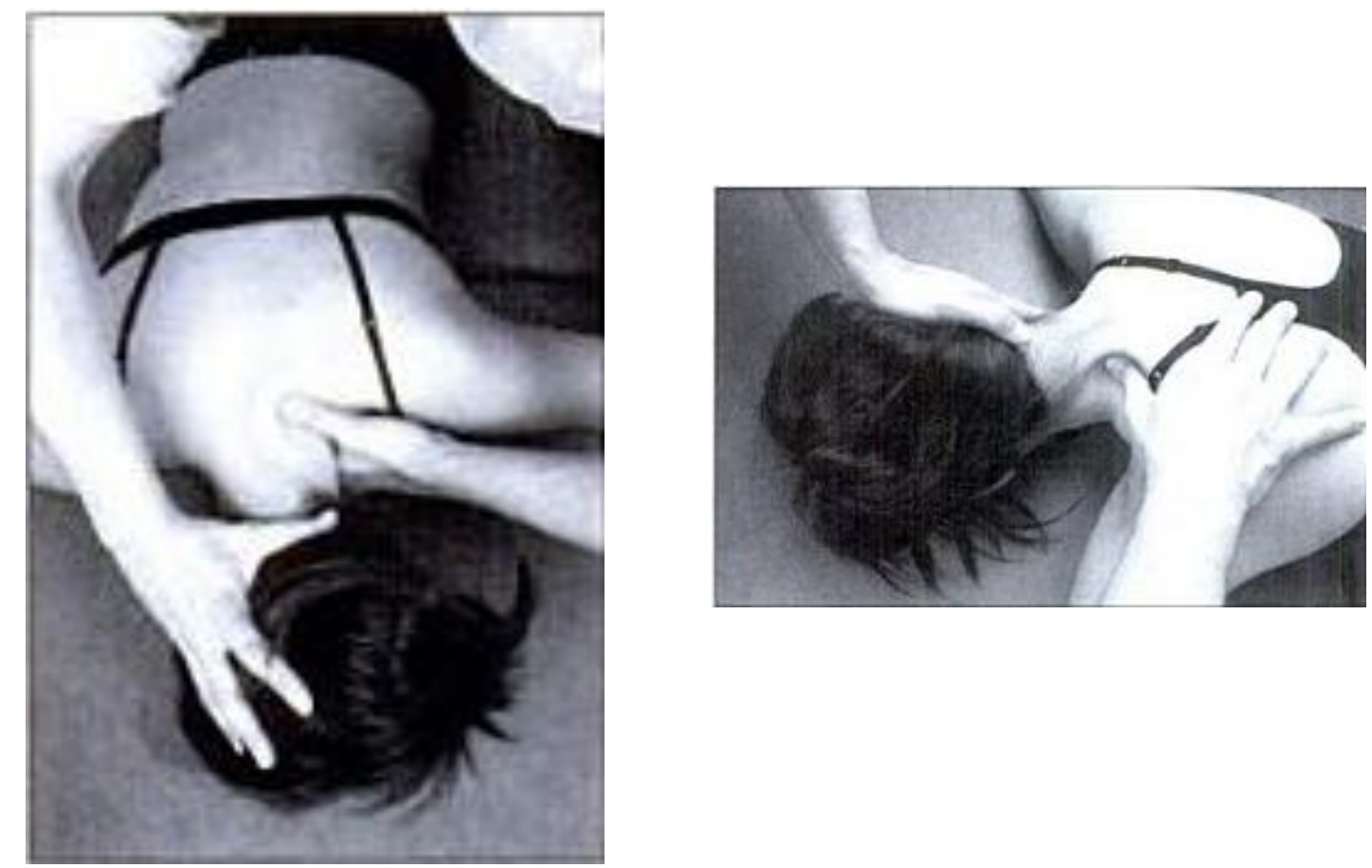

Figura 5: Procedimentos para manobra de manipulação de $\mathrm{C} 7$ sobre T1, em rotação direita e inclinação direita (RdSd), vista de dois ângulos diferentes. Figura adaptada de (CUECO, 2008).

\subsection{Processamento e Análise dos Sinais}

Todos os sinais adquiridos durante os experimentos foram processados por meio de uma rotina desenvolvida em ambiente MatLab (MathWorks, Inc. Natick, MA). Os sinais da plataforma de força foram convertidos por um sistema LabView a uma taxa de amostragem de $3 \mathrm{kHz}$. Os dados cinemáticos foram adquiridos a uma frequência de $100 \mathrm{~Hz}$. 


\subsubsection{Sinais da plataforma de Força (cinética)}

Para o cálculo do CoP nas direções ântero-posterior (AP) e médio-lateral (ML), foi realizada a calibração e conversão dos sinais adquiridos pela plataforma de força em unidades de força (N) e momento $\left(\mathrm{N}^{*}\right.$ metro). Feito isso, os sinais foram filtrados a uma frequência de corte de $8 \mathrm{~Hz}$ (filtro digital passa baixa tipo Butterworth de $4^{\mathrm{a}}$ ordem). Depois de filtrados, os primeiros $9 \mathrm{~s}$ e o último segundo correspondentes aos dados de cada repetição foram descartados, eliminando assim transientes devido à adaptação dos sujeitos e ao filtro digital aplicado. Assim, para os $50 \mathrm{~s}$ de sinais analisados, o "offset" dos dados do CoP foi removido usando-se a função "detrend" do Matlab, sendo possível então o cálculo do CoP nas duas direções (i.e., AP e ML) do seguinte modo:

$$
\begin{array}{ll}
\text { CoPap }=(-\mathrm{h} * \mathrm{FX}-\mathrm{MY}) / \mathrm{FZ} ; & \text { Equação 1 } \\
\mathrm{CoPml}=(-\mathrm{h} * \mathrm{FY}+\mathrm{MX}) / \mathrm{FZ} ; & \text { Equação 2 }
\end{array}
$$

onde h é a altura da base de apoio acima da plataforma de força, e Fx, Fy, Mx, My são, respectivamente as forças $(\mathrm{em} \mathrm{N})$ e os momentos (em N*metros) nas direções AP e ML; e Fz a força na direção vertical.

Finalmente as variáveis de interesse foram obtidas para os $50 \mathrm{~s}$ de sinais do CoP disponíveis para análise, sendo elas: 1) a área do estabilograma (Área), calculada a partir da obtenção de uma elipse englobando $95 \%$ dos dados do CoP, utilizando método proposto por Oliveira e colaboradores (1996); 2) a velocidade média (VM), calculada dividindo-se deslocamento total do CoP (em mm) pelo tempo total (em s), tanto na direção AP quanto ML; e 3) o valor eficaz (RMS), equivalente ao desvio padrão do deslocamento do CoP, também calculado tanto para a direção AP quanto ML. 


\subsubsection{Sinais do sistema de aquisição de imagens (cinemática)}

Os dados dos emissores posicionados no tornozelo, joelho, quadril e ombro foram usados para estimar a trajetória do centro de massa (COM) nos eixos AP e ML (COMap e COMml, respectivamente). Tal cálculo foi baseado em um modelo de três segmentos representando cabeçabraço-tronco (HAT), coxa e perna (WINTER, 1990). A localização do COM de cada seguimento foi determinada de acordo com medidas antropométricas apresentadas por Winter e colaboradores (WINTER, 1990), e o COM total do corpo foi então calculado como uma soma ponderada do COM de cada segmento. Uma vez computado o sinal do COM, variáveis análogas àquelas descritas para o sinal do CoP foram obtidas. Adicionalmente, tal disposição dos emissores possibilitou o cálculo dos seguintes ângulos segmentares (e a correspondente variação na posição angular em função do tempo): 1) $\theta$ Tornozelo; 2) $\theta$ Joelho e 3) $\theta$ Quadril, assim como representado na Figura 3.

\subsubsection{Análise Estatística}

Todas as análises estatísticas foram realizadas por meio do programa SPSS (Statistical Package for the Social Sciences Software Program for Windows, versão 18.0, Inc., Chicago, IL, EUA), com nível de significância de 95\% ( $\mathrm{p}<0,05)$. Primeiramente, a normalidade dos dados foi verificada através do teste de Shapiro-Wilk. Uma ANOVA de 2 vias (para medidas repetidas) com os repetição ( $1^{\mathrm{a}}$ a $10^{\mathrm{a}}$ repetição) e sessão experimental (EXP vs CTRL) foi utilizada para verificar efeitos e interações significativas entre os fatores. Quando apropriado, comparações múltiplas foram realizadas usando o pós-teste de Tukey. 


\section{RESULTADOS}

As Figuras de 6 a 10 apresentam valores das medidas extraídas do CoP. Os quadrantes superiores apresentam os valores de cada repetição para cada um dos 7 sujeitos analisados, em ambas as sessões experimentais (sessão EXP nos quadros da esquerda e sessão CTRL nos quadros da direita, como indicado em cada figura). Em cada quadro, os cinco primeiros valores correspondem ás tentativas (repetições) realizadas antes da manobra de manipulação, sendo as cinco tentativas subsequentes relativas às repetições realizadas após a manobra de manipulação. Cada sujeito está representado com uma cor diferente. Nos quadrados inferiores, os símbolos representam a média e o desvio padrão computados dentro da amostra $(n=7)$. Para facilitar a visualização, os valores estão normalizados em relação á média dos valores obtidos durante as 5 tentativas anteriores á manipulação (diminuindo assim a variabilidade inter-indivíduos). Os resultados da análise estatística (ANOVA de duas vias) aplicada sobre os parâmetros do CoP estão representados na Tabela 1. 
Area EXP

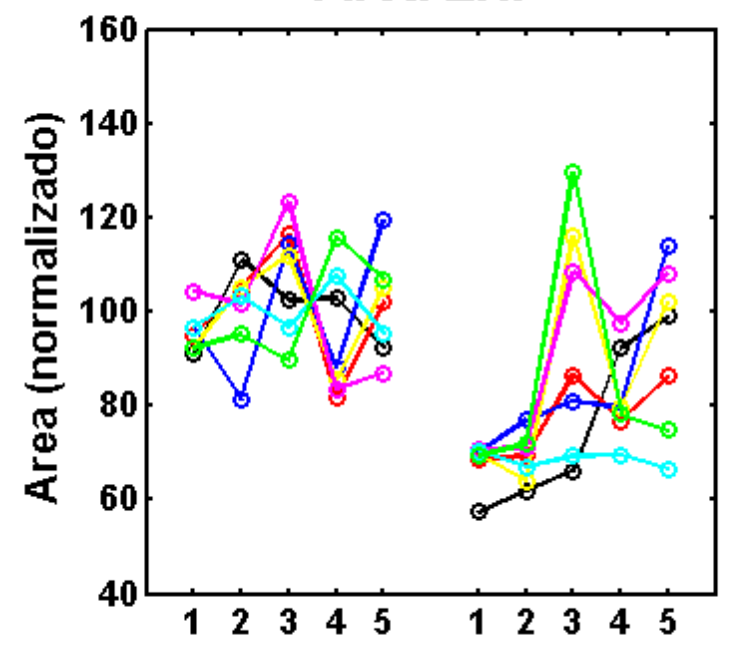

Area EXP

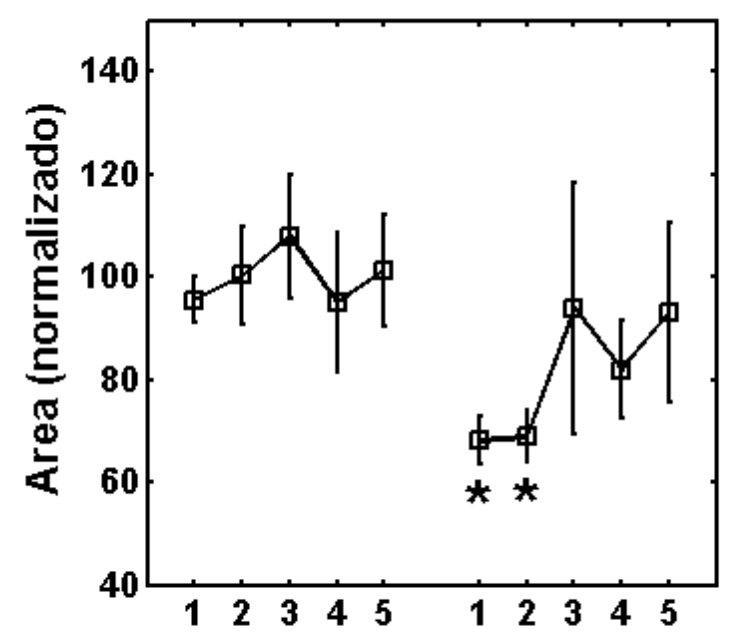

Area CTRL

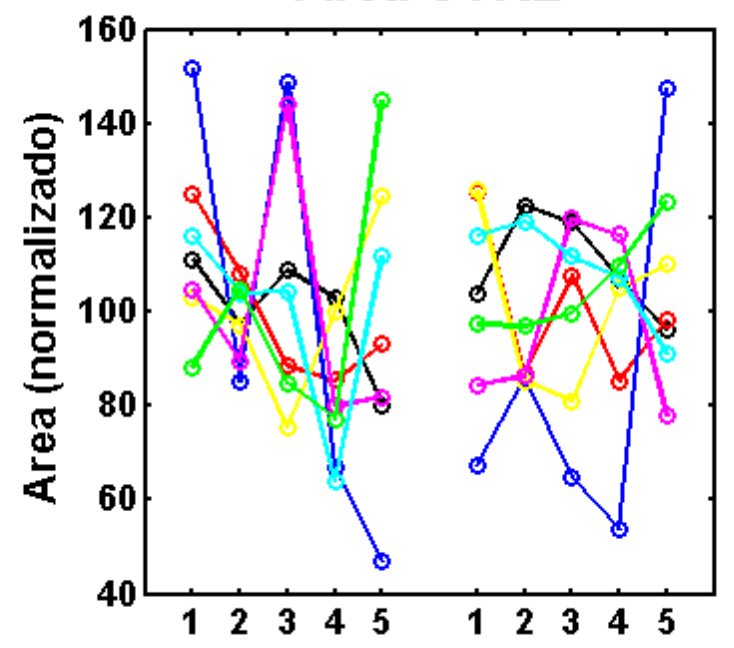

Area CTRL

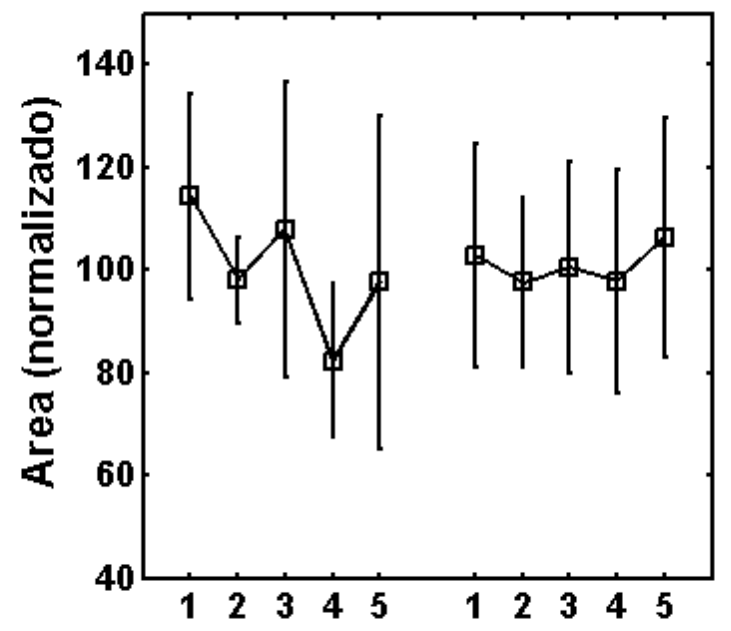

Figura 6: Quadrantes superiores representam os valores individuais (normalizados) para o parâmetro "Área" computado do sinal do CoP. Cada sujeito está representado com uma cor diferente. Em cada quadro, os cinco primeiros valores (á esquerda) correspondem ás tentativas realizadas antes da intervenção (manobra de manipulação cervical), enquanto os cinco valores posteriores (à direita) correspondem ás tentativas realizadas após a intervenção. O quadro da esquerda mostra valores obtidos durante a sessão experimental (EXP), na qual a manobra de manipulação cervical foi realizada, enquanto o quadro da direita representa a sessão controle (CTRL), na qual uma intervenção "placebo" foi aplicada. Nos quadrantes inferiores, os símbolos representam a média do grupo e as linhas verticais representam o desvio padrão amostra $(n=7)$. Asteriscos indicam diferenças significativas para a média observada nas repetições indicadas em comparação às demais. 

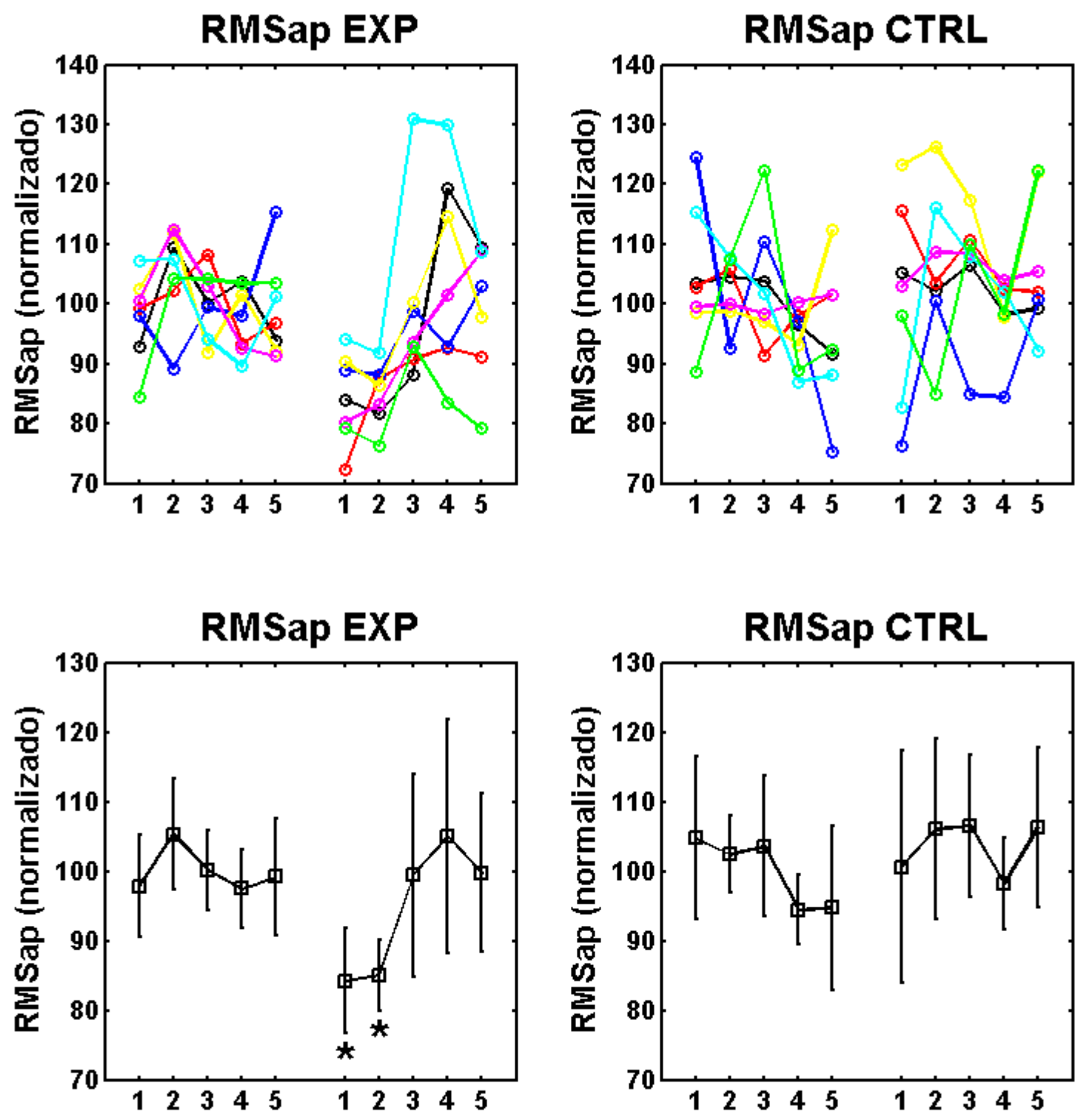

Figura 7: Idêntico á Figura 6, porém para o parâmetro "RMSap" computado do sinal do CoP. 

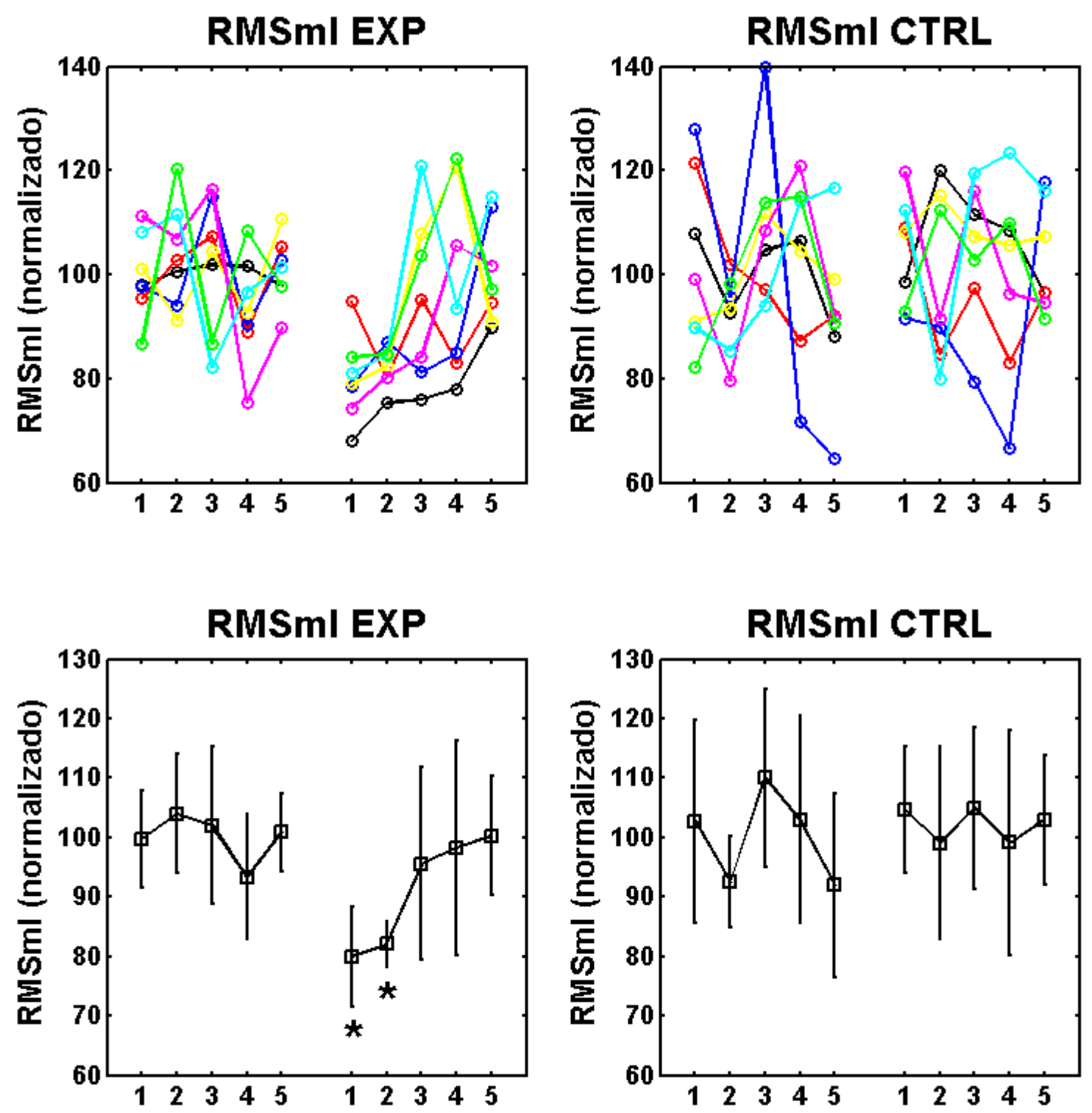

Figura 8: Idêntico á Figura 6, porém para o parâmetro "RMSml" computado do sinal do CoP. 

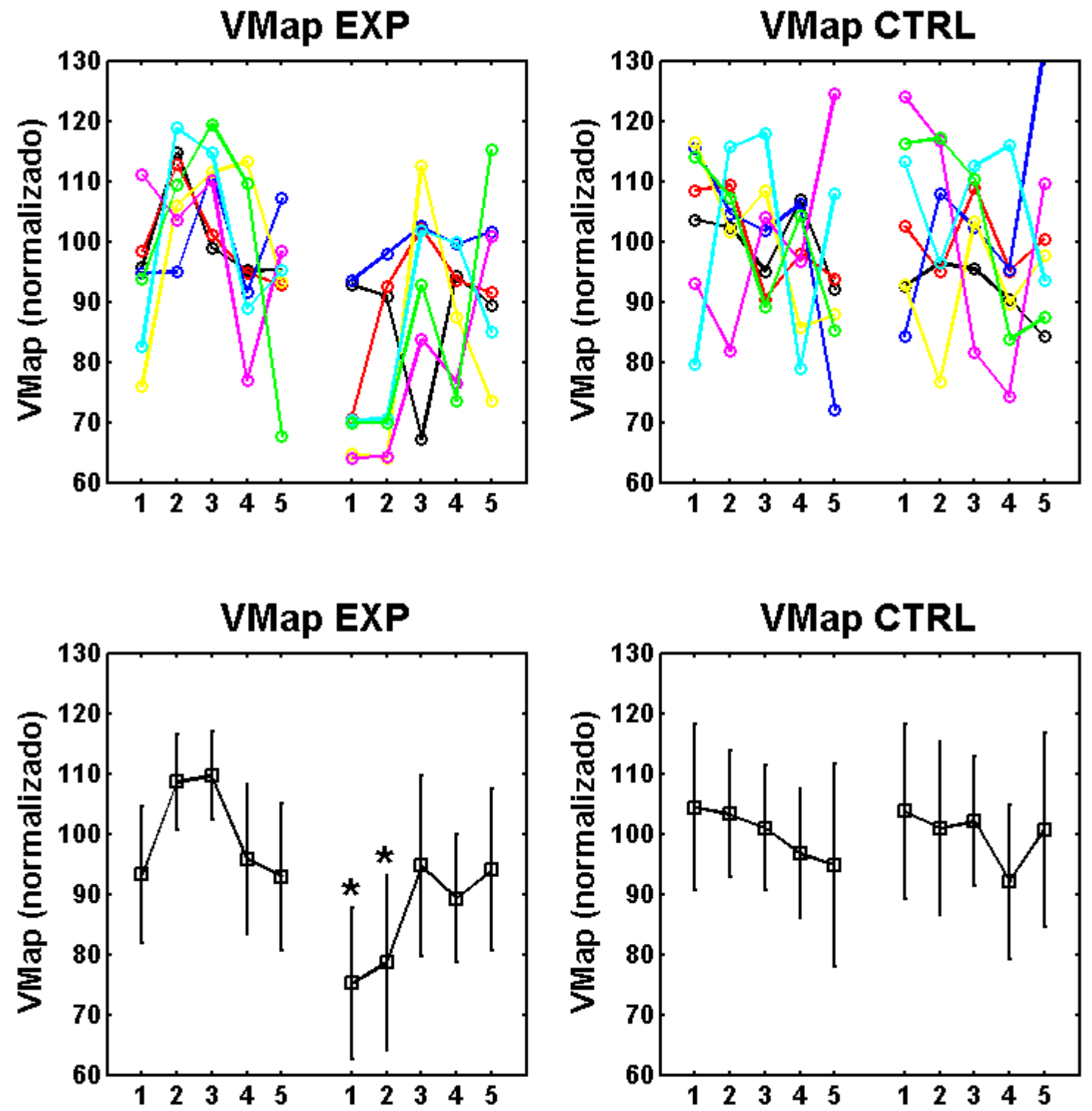

Figura 9: Idêntico á Figura 6, porém para o parâmetro "VMap" computado do sinal do CoP. 

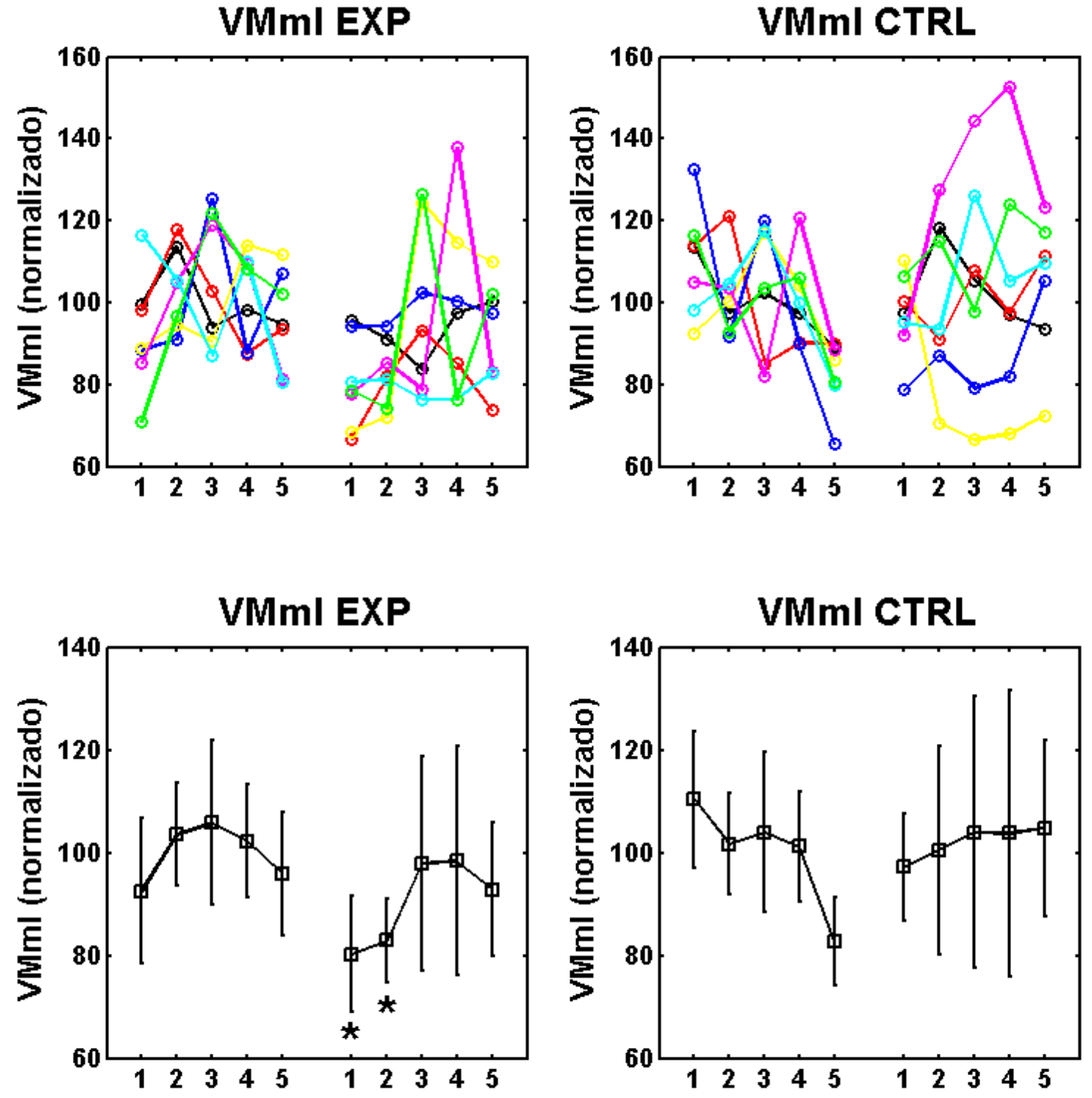

Figura 10: Idêntico á Figura 6, porém para o parâmetro "VMml" computado do sinal do CoP. 
Tabela 1: Resultados da ANOVA de duas vias (medidas repetidas) para os parâmetros do CoP.

\begin{tabular}{ccccccc}
\hline & \multicolumn{2}{l}{ Efeito do fator Repetição } & \multicolumn{2}{l}{ Efeito do fator Sessão } & \multicolumn{2}{c}{ Interação Repetição e Sessão } \\
& & $\mathrm{P}$ & $\mathrm{F}_{(1,6)}$ & $\mathrm{P}$ & $\mathrm{F}_{(9,6)}$ & $\mathrm{P}$ \\
Área do CoP & 2,672 & 0,012 & 12,882 & 0,012 & 2,589 & 0,015 \\
RMSap do CoP & 2,154 & 0,04 & 3,380 & 0,116 & 3,145 & 0,004 \\
RMSml do CoP & 1,340 & 0,239 & 8,632 & 0,026 & 3,054 & 0,005 \\
VMap do CoP & 2,679 & 0,012 & 23,139 & 0,003 & 2,088 & 0,022 \\
VMml do CoP & 2,432 & 0,021 & 2,242 & 0,185 & 3,078 & 0,005 \\
\hline
\end{tabular}

Pode-se notar que, para todas as variáveis associadas ao sinal do CoP (i.e. Área, RMSap, RMSml, VMap e VMml), houve um efeito significante de interação entre as fatores "repetição" e "sessão", indicando uma dependência da sessão (EXP ou CTRL) para o efeito da repetição (10 repetições, sendo 5 antes e 5 após o procedimento de manipulação) ou vice versa. Os testes post hoc indicaram que essa interação significante foi devido ao fato de que, nas sessões EXP, as repetições 6 e 7 (correspondentes as primeiras 2 tentativas pós-manipulação) apresentaram valores reduzidos em comparação ás demais repetições $(p<0,05)$, o que não ocorreu nas sessões CTRL visto que não foi encontra nenhuma diferença significante entre as sessões. Em outras palavras, na sessão EXP houve uma redução dos valores durante as primeiras 2 tentativas pós-manipulação em comparação ás tentativas pré-manipulação, sendo que após e terceira tentativa pós-manipulação houve uma tendência dos parâmetros voltarem a apresentar valores similares aqueles obtidos na situação prémanipulação. O mesmo não ocorreu na sessão CTRL, já que os parâmetros apresentaram uma tendência de permanecerem constantes (flutuando em torno de um valor médio) ao longo de toda sessão experimental. 
Resultados qualitativamente idênticos aos descrito para os parâmetros do CoP foram observados em relação às variáveis do COM. Por essa razão, as representações gráficas dos parâmetros extraídos dos sinais do COM não serão mostradas aqui. Os resultados da análise estatística (ANOVA de duas vias) aplicada sobre os parâmetros do COM estão representados na Tabela 2.

Tabela 2: Resultados da ANOVA de duas vias (medidas repetidas) para os parâmetros do COM.

\begin{tabular}{ccccccc}
\hline & \multicolumn{2}{l}{ Efeito do fator Repetição } & \multicolumn{2}{l}{ Efeito do fator Sessão } & \multicolumn{2}{l}{ Interação Repetição e Sessão } \\
\hline & $\mathrm{F}(9,6)$ & $\mathrm{P}$ & $\mathrm{F}(1,6)$ & $\mathrm{P}$ & $\mathrm{F}(9,6)$ & $\mathrm{P}$ \\
Área do COM & 1,581 & 0,145 & 12,494 & 0,12 & 2,509 & 0,018 \\
\hline RMSap do COM & 1,336 & 0,241 & 19,369 & 0,005 & 3,719 & 0,011 \\
RMSml do COM & 3,092 & 0,005 & 6,457 & 0,044 & 5,849 & $<0,001$ \\
VMap do COM & 3,404 & 0,002 & 0,379 & 0,561 & 2,691 & 0,013 \\
VMml do COM & 3,208 & 0,004 & 9,353 & 0,022 & 2,008 & 0,024 \\
\hline
\end{tabular}

As Figuras de 11 a 13 apresentam valores da variação da posição angular da articulação do tornozelo ( $\theta$ Tornozelo), do joelho ( $\theta$ Joelho) e do quadril ( $\theta$ Quadril). Nessas figuras, a representação gráfica segue o mesmo esquema utilizado na apresentação dos dados relativos aos parâmetros do CoP. Os resultados da análise estatística (ANOVA de duas vias) aplicada sobre os parâmetros de variação angular estão representados na Tabela 3. Nota-se que, para a $\theta$ Tornozelo, houve um efeito significante de interação entre os fatores "repetição" e "sessão", indicando uma dependência da sessão para o efeito da repetição (e vice versa). Semelhantemente ao que foi obsevado para os parâmetros do $\mathrm{CoP}$ e do $\mathrm{COM}$, os testes post hoc indicaram que essa interação foi associada à reduções significativas nas as repetições 6 e 7 (correspondentes as primeiras 2 tentativas pósmanipulação) em comparação às demais repetições, o que ocorreu apenas para a sessão EXP (não houve diferenças significantes entre as repetições para a sessão CTRL). Por outro lado, não foram observadas interações significantes entre os fatores "repetição" e "sessão" para os parâmetros $\theta$ Joelho 
e $\theta$ Quadril, mas sim um efeito significante apenas para o fator "sessão". Esses resultados indicam que, de uma maneira geral, os valores de $\theta$ Joelho e $\theta$ Quadril foram menores na sessão EXP em comparação à sessão CTRL, porém sem uma dependência da repetição (i.e. ordem da tentativa) realizada.
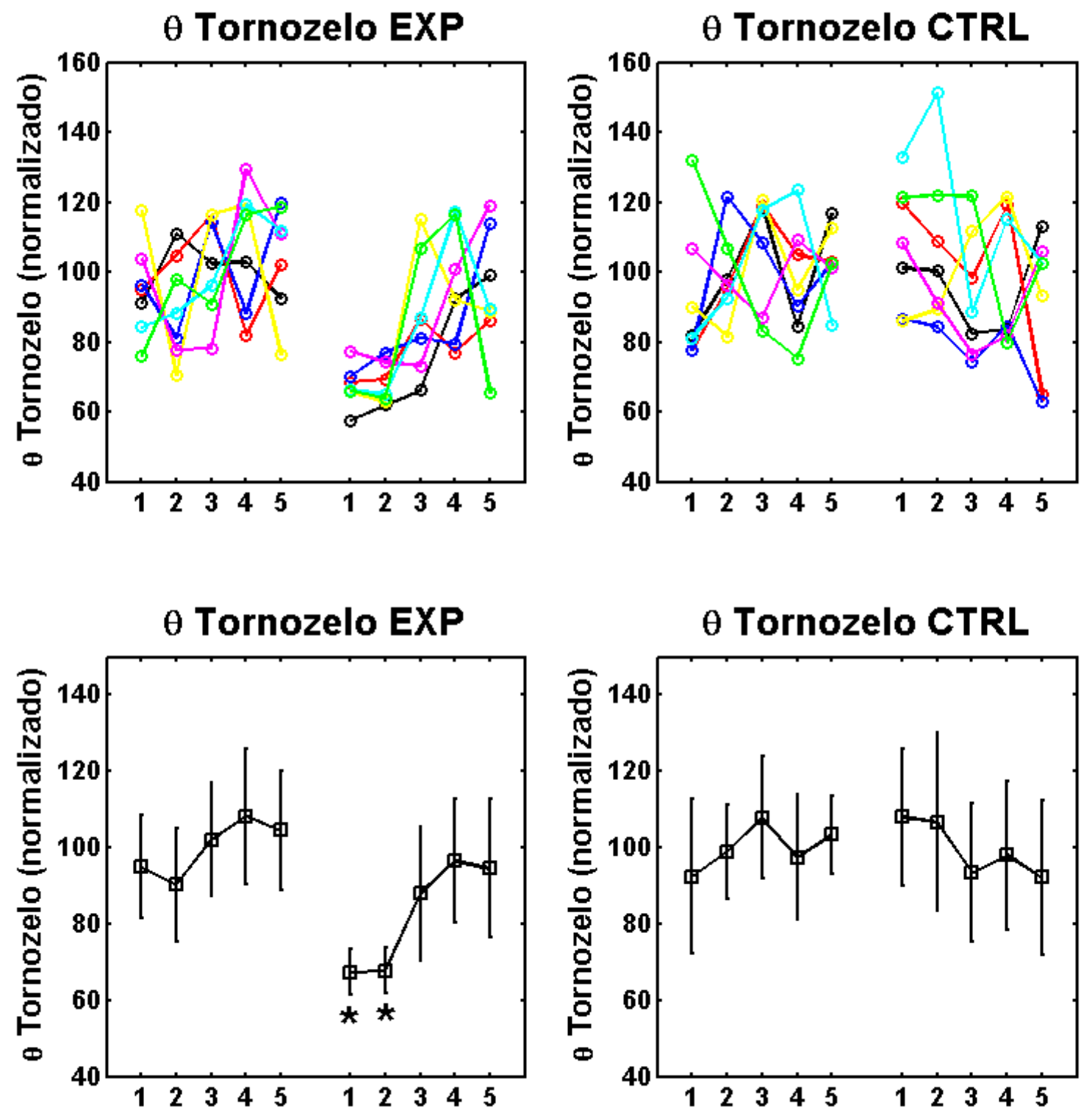

Figura 11: Idêntico á Figura 6, porém para o parâmetro " $\theta$ Tornozelo" computado a partir dos sinais da posição dos marcadores (cinemática). 

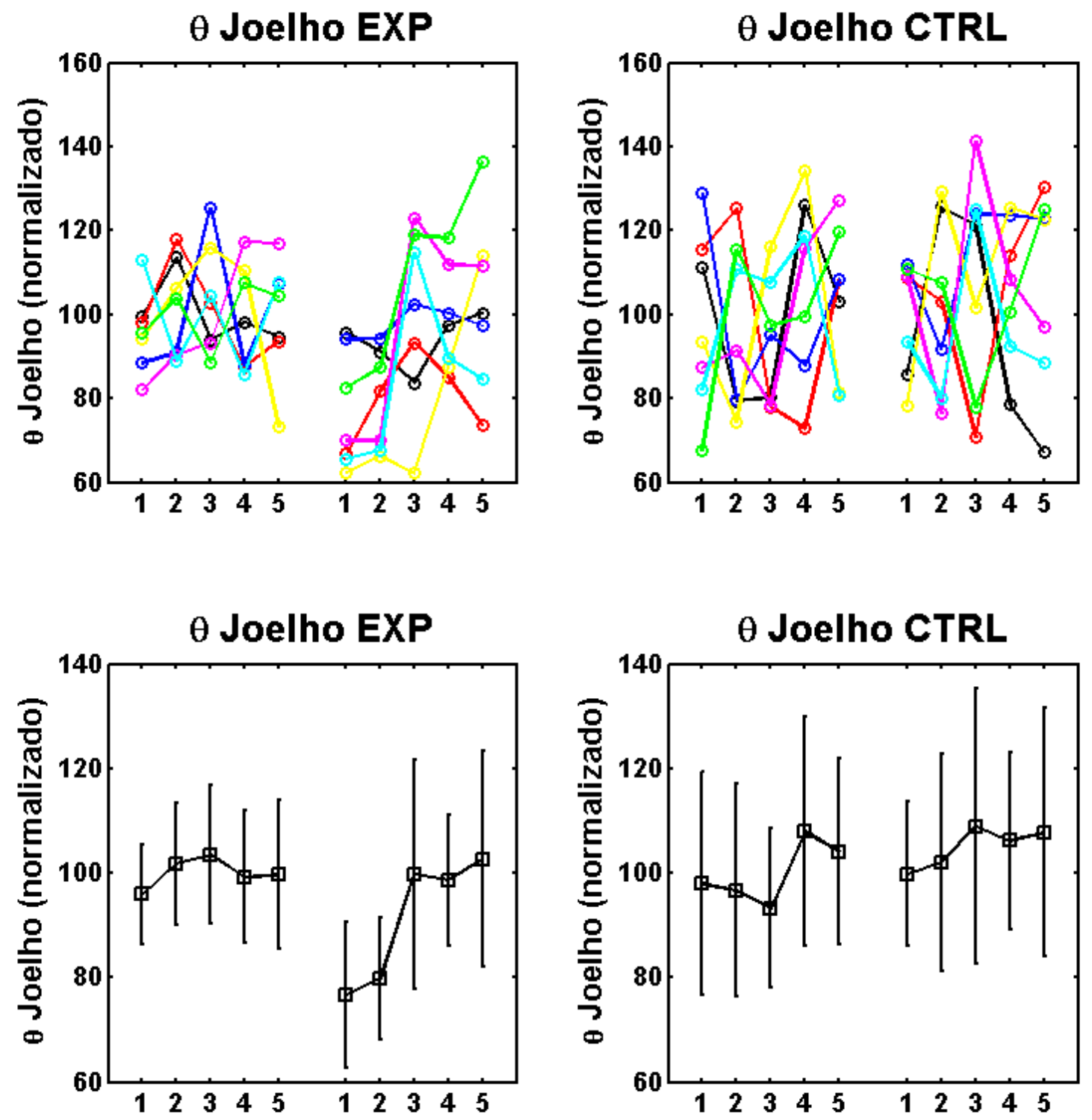

Figura 12: Idêntico á Figura 6, porém para o parâmetro " $\theta$ Joelho" computado a partir dos sinais da posição dos marcadores (cinemática). 

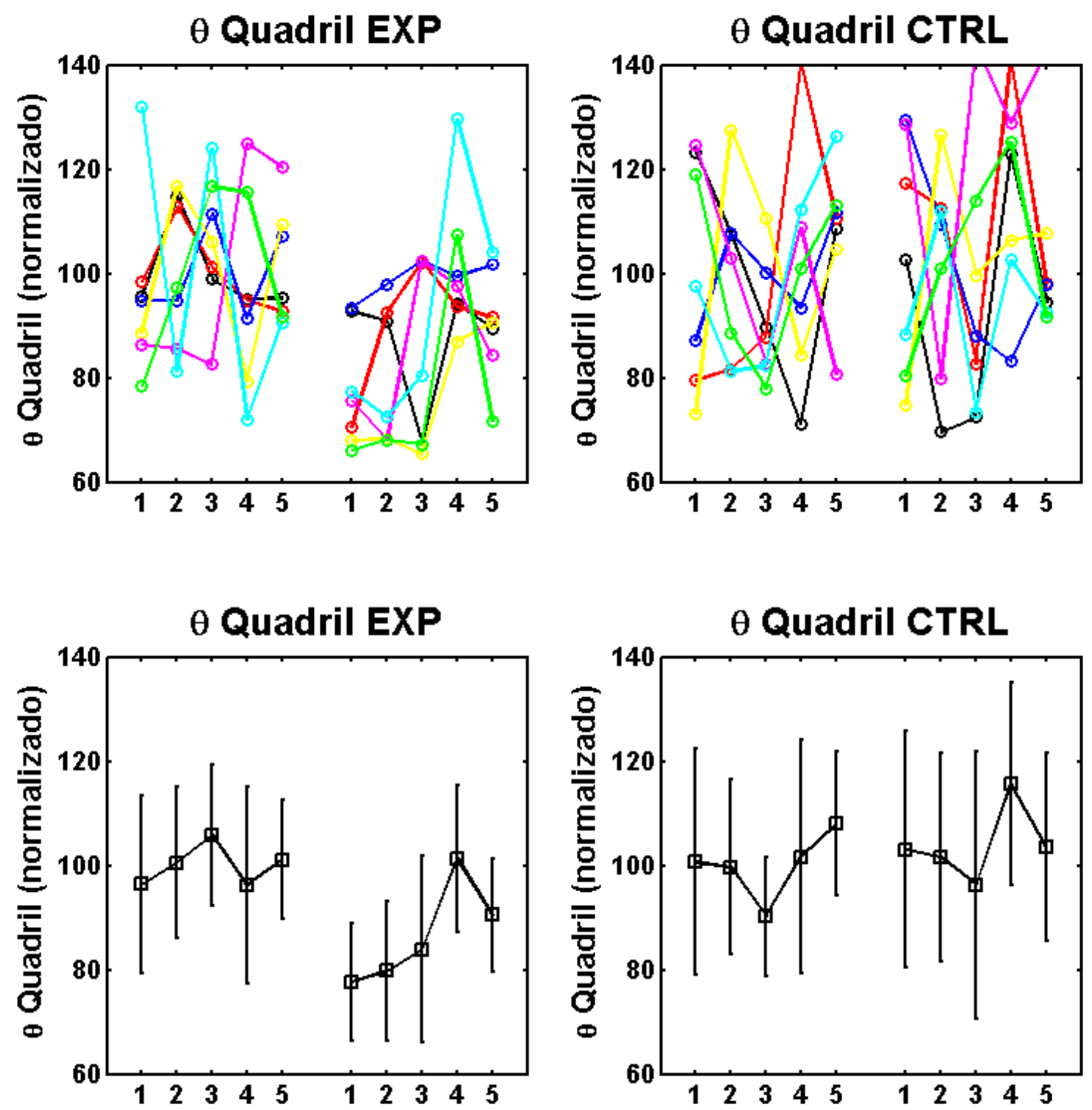

Figura 13: Idêntico á Figura 6, porém para o parâmetro " $\theta$ Joelho" computado a partir dos sinais da posição dos marcadores (cinemática). 
Tabela 3: Resultados da ANOVA de duas vias (medidas repetidas) para os parâmetros de variação nas posições angulares ( $\theta$ Tornozelo, $\theta$ Joelho e $\theta$ Quadril).

\begin{tabular}{|c|c|c|c|c|c|c|}
\hline & \multicolumn{2}{|c|}{ Efeito do fator Repetição } & \multicolumn{2}{|c|}{ Efeito do fator Sessão } & \multicolumn{2}{|c|}{ Interação Repetição e Sessão } \\
\hline & $\mathrm{F}(9,6)$ & $\mathrm{P}$ & $\mathrm{F}(1,6)$ & $\mathrm{P}$ & $\mathrm{F}(9,6)$ & $\mathrm{P}$ \\
\hline 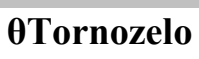 & 2,025 & 0,048 & 10,752 & 0,017 & 4,296 & $<0,001$ \\
\hline ӨJoelho & 1,164 & 0,336 & 7,515 & 0,034 & 1,498 & 0,176 \\
\hline$\theta Q u a d r i l$ & 1,675 & 0,118 & 9,893 & 0,020 & 1,614 & 0,135 \\
\hline
\end{tabular}




\section{DISCUSSÃO}

Os resultados do presente estudo sugerem que houve um efeito agudo da manobra de manipulação cervical. Ou seja, a manobra de manipulação gerou um efeito transitório, beneficiando o sistema de controle postural durante as primeiras 2 (ou 3, no caso de alguns sujeitos) tentativas realizadas após a intervenção. Após esse período, o efeito tende a desaparecer gradualmente, com o sistema de controle postural voltando a apresentar desempenho semelhante àquele observado antes da manobra de manipulação ter sido realizada. Nas sessões CTRL, nas quais os sujeitos foram submetidos a uma intervenção "placebo", ou seja, sem que a manobra de manipulação fosse completada, o mesmo efeito não foi observado, já que o sistema de controle postural se mostrou estável durante todo o experimento.

Efeitos transitórios sobre o sistema de controle postural foram reportados anteriormente em resposta a estímulos vibratórios, á fadiga muscular, à estimulação vestibular galvânica, entre outros (DAY et al., 1997; EKLUND, 1972; PAILLARD, 2012; WIERZBICKA et al., 1998). No presente estudo, o efeito agudo é interpretado como resultado de alterações transitórias nas estruturas cervicais decorrentes da manobra de manipulação. Mais especificamente, especula-se que a manobra de manipulação tenha causado liberação de estruturas cervicais como tecidos moles, ossos e vasos sanguíneos, favorecendo assim o influxo de informação sensorial relevante para o sistema de controle postural, como comentado na Introdução. Esse efeito, no entanto, tende a desaparecer já a partir da terceira tentativa pós-manipulação (o que nos presentes experimentos representa um tempo de aproximadamente 5 minutos), possivelmente devido a uma reorganização das estruturas cervicais após a manobra, ou ainda a um fenômeno de adaptação/habituação por parte dos receptores sensoriais e estruturas envolvidas. Adicionalmente, um efeito direto da manipulação sobre o aparato vestibular não pode ser descartado, ou seja, a rápida perturbação gerada pela manobra de manipulação pode ter atuado, diretamente por efeito mecânico, sobre as estruturas vestibulares que exercem importante influência no controle da tarefa postural. 
È importante ressaltar, no entanto, que não é possível avaliar de forma precisa os mecanismos específicos associados aos efeitos observados no presente estudo. Diversas possibilidades podem ser apontadas, como modificação na transmissão de informações sensoriais por parte: de receptores musculares (e.g. fusos neuromusculares, órgão tendinosos de Golgi) (ABRAHAMS, 1977; BOYDCLARK et al., 2002; KULKARNI et al., 2001; LIU et al., 2003); de receptores articulares (e.g. terminais de Ruffini, corpúsculos pacinianos, receptores ligamentares) (MCLAIN, 1994); do aparato vestibular (seja diretamente por estímulo mecânico ou indiretamente por liberação do aporte sanguíneo causado por mudanças nas estruturas vasculares) (BOLTON, 1998; NITZ; PECK, 1986); e de mecanismos associados à função do sistema nervoso central (já que pode ter ocorrido, por exemplo, mudanças no fluxo sanguíneo para regiões espinhais, subcorticais e corticais) (SHUMWAY-COOK; WOOLLACOTT, 2001). Adicionalmente, vale a pena citar que na região cervical onde foi realizada a manobra de manipulação (C7 - T1) fica localizado o gânglio cervicotorácico (ou gânglio estrelado), que atua no controle simpático de vasodilatação/vasocontrição das artérias vertebrais (HUNTOON, 2010) mencionadas na Introdução. Portanto, uma redução na atividade deste gânglio (supostamente gerada pela manipulação dos tecidos adjacentes) pode ter favorecido a fluxo sanguíneo para regiões cerebrais (JAIN et al., 2011) e vestibulares (GALETTI et al., 1969; SOKOLOWSKI, 1962), auxiliando momentaneamente estruturas importantes para sistema de controle postural. Tendo em vista os vários fatores especulados aqui, existe uma clara necessidade de estudos futuros, possivelmente envolvendo técnicas de microneurografia (a fim de se monitorar os disparos de vias sensoriais e motoras específicas) ou técnicas de imageamento (a fim de avaliar a ativação de diferentes partes do sistema nervoso ou fluxo sanguíneo), para que os mecanismos associados aos presentes resultados possam ser adequadamente compreendidos.

Foram observados efeitos semelhantes para as variáveis extraídas dos sinais do CoP e do COM, isto é, tanto os parâmetros clássicos de controle postural medidos através da plataforma de força quanto através da cinemetria refletiram de forma semelhante uma redução das oscilações posturais durante as 2 primeiras tentativas após a manipulação. Durante tarefas de controle postural, 
as componentes de baixa frequência do $\mathrm{CoP}$ (por exemplo, até $0.4 \mathrm{~Hz}$ ) têm sido fortemente associadas às oscilações na trajetória do COM (BENDA et al., 1994; CARON et al., 1997; GAGE et al., 2004). No entanto, o sinal do CoP também apresenta componentes de frequências mais altas (por exemplo, de 0.5 até $2 \mathrm{~Hz}$ ) que não estão presentes no sinal do COM. Tais componentes de alta frequência do CoP representam contrações musculares ou pequenos movimentos de outros segmentos do corpo distantes ao tornozelo. Sendo assim, na hipótese de um efeito específico da manipulação cervical sobre essas contrações musculares ou movimentos articulares (que eventualmente não se refletissem na estabilização do $\mathrm{COM}$ ), efeitos diferenciais entre os parâmetros do CoP e do COM teriam sido observados (i.e. presença de efeito na trajetória CoP e ausência na trajetória do COM). Portanto, o comportamento semelhante entre os parâmetro do CoP e do COM sugere que a manobra de manipulação cervical levou a estabilização postural do COM (também refletida nos sinais do CoP) sem um efeito significante sobre contrações musculares e movimentações articulares distantes do tornozelo. Os resultados observados para variação da posição angular das articulações do tornozelo ( $\theta$ Tornozelo), do joelho ( $\theta$ Joelho) e do quadril ( $\theta$ Quadril) reforçam essa perspectiva. Mais especificamente, a ausência de efeitos significantes entre as repetições, assim como a ausência de interações significantes entre os fatores "repetição" e "sessão" para os parâmetros $\theta$ Joelho e $\theta$ Quadril sugere que a manobra de manipulação deu origem a uma estabilização postural principalmente em decorrência de uma diminuição nas flutuações da posição angular dna articulação do tornozelo (tendo que em vista que o parâmetro $\theta$ Tornozelo mostrou efeitos semelhantes aos do CoP e do COM) . Em outras palavras, apesar de torques gerados nas articulações do tornozelo, joelho e quadril serem capazes de atuar na estabilização da postura (YANG et al., 1990), os presentes achados sugerem uma estabilização do sistema de controle postural atuando como um modelo de pêndulo invertido com estratégia centralizada na articulação tornozelo (LORAM et al., 2001).

Os experimentos reportados aqui foram conduzidos de maneira que aproximadamente metade dos participantes (4 sujeitos) realizaram a sessão EXP antes da sessão CTRL, enquanto os restantes (3 sujeitos) realizaram a sessão CTRL primeiro. Sendo assim, é improvável que um efeito de 
prática/habituação em relação aos procedimentos experimentais tenha influenciado no resultado, visto que os efeitos observados não estiveram presentes na sessão CTRL, mas apenas na sessão EXP, Além disso, dentro das mesmas sessões experimentais, não foram observados tendências lineares de mudanças no desempenho do sistema de controle postural ao longo do tempo. Devido ao número amostral de 7 sujeitos, é possível que uma análise estatística incluindo dados de sujeitos adicionais forneça resultados mais consistentes, confirmando de forma mais segura as tendências relatadas no presente estudo. No entanto, apesar dos estudos que investigam efeitos de intervenções específicas sobre controle postural humano geralmente envolverem um número amostral maior do que o utilizado aqui [tipicamente entre 10 e 20 sujeitos (HOCH; RUSSELL, 2016)], a potência estatística associada aos testes empregados no presente estudo mostrou valores sempre superiores a 0.89 , indicando que os efeitos reportados são bastante confiáveis (COHEN, 1988). 


\section{CONCLUSÃO}

A manobra de manipulação cervical causou uma redução das oscilações posturais na tarefa de equilíbrio unipodal durante as primeiras 2 tentativas realizadas após a intervenção, refletindo portanto um efeito agudo e transitório da manobra sobre o sistema de controle postural. Após um período de aproximadamente 5 minutos, esse efeito tende a desaparecer gradualmente, com o sistema de controle postural voltando a apresentar desempenho semelhante àquele observado antes da manobra de manipulação. Ainda, os resultados sugerem que a manobra de manipulação deu origem a uma estabilização associada principalmente a uma diminuição nas flutuações da posição angular da articulação do tornozelo, visto que não foram encontrados significantes sobre as variação na posição angular do joelho e do quadril. Estudos futuros são necessários para que os mecanismos específicos associados aos presentes resultados possam ser adequadamente compreendidos. 


\section{REFERÊNCIAS}

ABRAHAMS, V. C. The physiology of neck muscles; their role in head movement and maintenance of posture. Can J Physiol Pharmacol, 55, 3 332-8, 1977.

ALBURQUERQUE-SENDIN, F. et al. Immediate effects of bilateral manipulation of talocrural joints on standing stability in healthy subjects. Man Ther, 14, 1 75-80, 2009.

ANDARY, M. T. et al. Neurogenic atrophy of suboccipital muscles after a cervical injury: a case study. Am J Phys Med Rehabil, 77, 6 545-9, 1998.

BENDA, J. B. et al. Biomechanical relationship between center of gravity and center of pressure during standing. IEEE Trans Rehabil Eng, 2, 1 3-10, 1994.

BIEMOND, A.; DE JONG, J. M. On cervical nystagmus and related disorders. Brain, 92, 2 437-58, 1969.

BOLTON, P. S. The somatosensory system of the neck and its effects on the central nervous system. J Manipulative Physiol Ther, 21, 8 553-63, 1998.

BOTTARO, A. et al. Body sway during quiet standing: is it the residual chattering of an intermittent stabilization process? Human Movement Science, 24, 588-615, 2005.

BOVE, M. et al. Interaction between vision and neck proprioception in the control of stance. Neuroscience, 164, 4 1601-8, 2009.

BOYD-CLARK, L. C. et al. Muscle spindle distribution, morphology, and density in longus colli and multifidus muscles of the cervical spine. Spine (Phila Pa 1976), 27, 7 694-701, 2002.

BRANDT, T.; BRONSTEIN, A. M. Cervical vertigo. Journal of neurology, neurosurgery, and psychiatry, 71, 1 8-12, 2001.

CAPICIKOVA, N. et al. Human postural response to lower leg muscle vibration of different duration. Physiological research / Academia Scientiarum Bohemoslovaca, 55 Suppl 1, S129-34, 2006.

CARON, O. et al. Estimating the centre of gravity of the body on the basis of the centre of pressure in standing posture. J Biomech, 30, 11-12 1169-71, 1997.

CEYTE, H. et al. Effects of neck muscles vibration on the perception of the head and trunk midline position. Experimental brain research. Experimentelle Hirnforschung, 170, 1 136-40, 2006.

COHEN, J. In: Statistical power analysis for the behavioral sciences. Hillsdale, NJ: Lawerence Erlbaum Associates, 1988.

COHEN, L. A. Role of eye and neck proprioceptive mechanisms in body orientation and motor coordination. Journal of neurophysiology, 24, 1-11, 1961.

COMAN, W. B. Dizziness relared to ENT conditions.In Grieve, G. P. Grieve's modern manual therapy of the vertebral column. Churchill Livingstone: Edinburgh, 1986.

COURJON, J. H. et al. Vestibular nerve and nuclei unit responses and eye movement responses to repetitive galvanic stimulation of the labyrinth in the rat. Experimental brain research. Experimentelle Hirnforschung, 66, 1 41-8, 1987. 
CUECO, R. T. In: La columna cervical: evaluación clínica y aproximaciones terapéuticas: Principios anatómicos y funcionales, exploración clínica y técnicas de tratemiento. Madrid: Médica Panamericana, 2008 .

DAY, B. L. et al. Human body-segment tilts induced by galvanic stimulation: a vestibularly driven balance protection mechanism. The Journal of physiology, 500 ( Pt 3), 661-72, 1997.

DE JONG, P. T. et al. Ataxia and nystagmus induced by injection of local anesthetics in the Neck. Annals of neurology, 1, 3 240-6, 1977.

DUARTE, M.; ZATSIORSKY, V. M. Long-range correlations in human standing. Physics Letters A, 283, 12 124-128, 2001.

DUARTE, M.; ZATSIORSKY, V. M. On the fractal properties of natural human standing. Neuroscience Letters, 283, 3 173-6, 2000.

EASTON, R. D. et al. Auditory cues for orientation and postural control in sighted and congenitally blind people. Experimental brain research. Experimentelle Hirnforschung, 118, 4 541-50, 1998.

EKLUND, G. General features of vibration-induced effects on balance. Upsala journal of medical sciences, 77, 2 112-24, 1972.

FERREIRA, M. L. et al. Changes in postural activity of the trunk muscles following spinal manipulative therapy. Man Ther, 12, 3 240-8, 2007.

FRAIX, M. Osteopathic manipulative treatment and vertigo: a pilot study. PM R, 2, 7 612-8, 2010.

FRAIX, M. et al. Use of the SMART Balance Master to quantify the effects of osteopathic manipulative treatment in patients with dizziness. J Am Osteopath Assoc, 113, 5 394-403, 2013.

GAGE, W. H. et al. Kinematic and kinetic validity of the inverted pendulum model in quiet standing. Gait \& posture, 19, 2 124-32, 2004.

GALETTI, G. et al. [Electronystagmorgraphic and ophthalmodynamographic findings after anesthetic block of stellate ganglion in patients with vertigo syndromes]. Minerva Otorinolaringol, 19, 2 75-86, 1969.

GALM, R. et al. Vertigo in patients with cervical spine dysfunction. Eur Spine J, 7, 1 55-8, 1998.

GOERTZ, C. M. et al. Effects of spinal manipulation on sensorimotor function in low back pain patients - A randomized controlled trial. Man Ther, 2015.

GOMEZ, S. et al. Differences between body movement adaptation to calf and neck muscle vibratory proprioceptive stimulation. Gait \& posture, 30, 1 93-9, 2009.

HALLGREN, R. C. et al. Atrophy of suboccipital muscles in patients with chronic pain: a pilot study. J Am Osteopath Assoc, 94, 12 1032-8, 1994.

HATTON, A. L. et al. The effect of textured surfaces on postural stability and lower limb muscle activity. J Electromyogr Kinesiol, 19, 5 957-64, 2009.

HAWK, C. et al. Chiropractic care for nonmusculoskeletal conditions: a systematic review with implications for whole systems research. J Altern Complement Med, 13, 5 491-512, 2007.

HAY, L. et al. Availability of visual and proprioceptive afferent messages and postural control in elderly adults. Experimental brain research. Experimentelle Hirnforschung, 108, 1 129-39, 1996. 
HEIKKILA, H. et al. Effects of acupuncture, cervical manipulation and NSAID therapy on dizziness and impaired head repositioning of suspected cervical origin: a pilot study. Man Ther, 5, 3 151-7, 2000.

HOCH, M. C.; RUSSELL, D. M. Plantar cooling does not affect standing balance: A systematic review and meta-analysis. Gait \& posture, 43, 1-8, 2016.

HUNTOON, M. A. The vertebral artery is unlikely to be the sole source of vascular complications occurring during stellate ganglion block. Pain Pract, 10, 1 25-30, 2010.

HWANG, S. et al. Dynamic reweighting of three modalities for sensor fusion. PloS one, 9, 1 e88132, 2014.

JAIN, V. et al. Stellate ganglion block for treatment of cerebral vasospasm in patients with aneurysmal subarachnoid hemorrhage - A preliminary study. J Anaesthesiol Clin Pharmacol, 27, 4 516-21, 2011.

JANSSENS, L. et al. Proprioceptive changes impair balance control in individuals with chronic obstructive pulmonary disease. PloS one, 8, 3 e57949, 2013.

JEKA, J. J. Light touch contact as a balance aid. Physical therapy, 77, 5 476-87, 1997.

JEKA, J. J.; LACKNER, J. R. Fingertip contact influences human postural control. Experimental brain research. Experimentelle Hirnforschung, 100, 3 495-502, 1994.

KARLBERG, M. et al. Postural and symptomatic improvement after physiotherapy in patients with dizziness of suspected cervical origin. Archives of physical medicine and rehabilitation, 77, 9 874-82, 1996.

KARNATH, H. O. Subjective body orientation in neglect and the interactive contribution of neck muscle proprioception and vestibular stimulation. Brain, 117 ( Pt 5), 1001-12, 1994.

KERRY, R.; TAYLOR, A. J. Cervical arterial dysfunction assessment and manual therapy. Man Ther, 11, 4 243-53, 2006.

KULKARNI, V. et al. Quantitative study of muscle spindles in suboccipital muscles of human foetuses. Neurol India, 49, 4 355-9, 2001.

LIU, J. X. et al. Muscle spindles in the deep muscles of the human neck: a morphological and immunocytochemical study. J Histochem Cytochem, 51, 2 175-86, 2003.

LOPEZ, D. et al. Effects of comprehensive osteopathic manipulative treatment on balance in elderly patients: a pilot study. J Am Osteopath Assoc, 111, 6 382-8, 2011.

LORAM, I. D. et al. Human balancing of an inverted pendulum: is sway size controlled by ankle impedance? The Journal of physiology, 532, Pt 3 879-91, 2001.

LYSTAD, R. P. et al. Manual therapy with and without vestibular rehabilitation for cervicogenic dizziness: a systematic review. Chiropr Man Therap, 19, 1 21, 2011.

MAGAlHAES, F. H.; KOHN, A. F. Vibratory noise to the fingertip enhances balance improvement associated with light touch. Experimental brain research. Experimentelle Hirnforschung, 209, 1 139-51, 2011.

MAGAREY, M. E. et al. Pre-manipulative testing of the cervical spine review, revision and new clinical guidelines. Man Ther, 9, 2 95-108, 2004.

MASANI, K. et al. Importance of body sway velocity information in controlling ankle extensor activities during quiet stance. Journal of Neurophysiology, 90, 3774-3782, 2003.

MAURER, C. et al. Multisensory control of human upright stance. Exp. Brain Res, 171, 231-250, 2005. 
MCLAIN, R. F. Mechanoreceptor endings in human cervical facet joints. Spine (Phila Pa 1976), 19, 5 495$501,1994$.

MCPARTLAND, J. M. et al. Chronic neck pain, standing balance, and suboccipital muscle atrophy--a pilot study. J Manipulative Physiol Ther, 20, 1 24-9, 1997.

MINOR, L. B.; GOLDBERG, J. M. Vestibular-nerve inputs to the vestibulo-ocular reflex: a functionalablation study in the squirrel monkey. J Neurosci, 11, 6 1636-48, 1991.

NITZ, A. J.; PECK, D. Comparison of muscle spindle concentrations in large and small human epaxial muscles acting in parallel combinations. Am Surg, 52, 5 273-7, 1986.

OLIVEIRA, L. F. et al. Calculation of area of stabilometric signals using principal component analysis. Physiological measurement, 17, 4 305-12, 1996.

PAILLARD, T. Effects of general and local fatigue on postural control: a review. Neurosci Biobehav Rev, 36, 1 162-76, 2012.

PATEL, M. et al. The effect of foam surface properties on postural stability assessment while standing. Gait $\boldsymbol{\&}$ posture, 28, 4 649-56, 2008.

PETERKA, R. J. Postural control model interpretation of stabilogram diffusion analysis. Biol. Cybern., 82, 335-343, 2000.

PRIETO, T. E. et al. Measures of postural steadiness: differences between healthy young and elderly adults. IEEE Trans Biomed Eng, 43, 956-966, 1996.

PRIPLATA, A. A. et al. Vibrating insoles and balance control in elderly people. Lancet, 362, 9390 1123-4, 2003.

REID, S. A.; RIVETT, D. A. Manual therapy treatment of cervicogenic dizziness: a systematic review. Man Ther, 10, 1 4-13, 2005.

SHUMWAY-COOK, A.; WOOLLACOTT, M. H. In: Motor control : theory and practical applications. Philadelphia ; London: Lippincott Williams \& Wilkins, 2001.

SOKOLOWSKI, S. [Effect of blocking of the stellate ganglion on vestibular reactions]. Otolaryngol Pol, 16, 99-104, 1962.

STANEK, J. M. et al. Single-limb-balance difficulty on 4 commonly used rehabilitation devices. J Sport Rehabil, 22, 4 288-95, 2013.

TRELEAVEN, J. Sensorimotor disturbances in neck disorders affecting postural stability, head and eye movement control--Part 2: case studies. Man Ther, 13, 3 266-75, 2008.

TRELEAVEN, J. et al. Comparison of sensorimotor disturbance between subjects with persistent whiplashassociated disorder and subjects with vestibular pathology associated with acoustic neuroma. Archives of physical medicine and rehabilitation, 89, 3 522-30, 2008.

TSANG, S. M. et al. Normal kinematics of the neck: the interplay between the cervical and thoracic spines. Man Ther, 18, 5 431-7, 2013.

WAPNER, S. et al. Experiments on sensory-tonic field theory of perception. I. Effect of extraneous stimulation on the visual perception of verticality. J Exp Psychol, 42, 5 341-4, 1951. 
WASSINGER, C. A. et al. Cervical \& thoracic manipulations: Acute effects upon pain pressure threshold and self-reported pain in experimentally induced shoulder pain. Man Ther, 2015.

WASSINGER, C. A. et al. Acute effects of rearfoot manipulation on dynamic standing balance in healthy individuals. Man Ther, 19, 3 242-5, 2014.

WATANABE, R. N. et al. Influences of premotoneuronal command statistics on the scaling of motor output variability during isometric plantar flexion. Journal of neurophysiology, 110, 11 2592-606, 2013.

WIERZBICKA, M. M. et al. Vibration-induced postural posteffects. Journal of neurophysiology, 79, 1 14350., 1998.

WINTER, D. A. In: Biomechanics and motor control of human movement. New York ; Chichester: Wiley, 1990.

WOOLLACOTT, M. H. et al. Aging and posture control: changes in sensory organization and muscular coordination. Int J Aging Hum Dev, 23, 2 97-114, 1986.

WU, G.; CHIANG, J. H. The significance of somatosensory stimulations to the human foot in the control of postural reflexes. Experimental brain research. Experimentelle Hirnforschung, 114, 1 163-9, 1997.

YANG, J. F. et al. Postural dynamics in the standing human. Biological cybernetics, 62, 4 309-20, 1990.

ZATSIORSKY, V. M.; DUARTE, M. Rambling and trembling in quiet standing. Motor Control, 4, 2 185200, 2000.

ZHOU, W. et al. Clinical study on manipulative treatment of derangement of the atlantoaxial joint. J Tradit Chin Med, 19, 4 273-8, 1999. 